Sharif University of Technology
Scientia Iranica
Transactions E: Industrial Engineering
hCIENTIA

\title{
Three-valued soft set and its multi-criteria group decision making via TOPSIS and ELECTRE
}

\author{
E. Akçetin ${ }^{a}$ and H. Kamacı ${ }^{b, *}$ \\ a. Department of Accounting and Financial Management, Seydikemer School of Applied Sciences, Mug̃la Sıtkı Koçman University, \\ Mug̃la, Turkey. \\ b. Department of Mathematics, Faculty of Science and Arts, Yozgat Bozok University, Yozgat, Turkey.
}

Received 24 October 2019; received in revised form 3 January 2020; accepted 20 April 2020

\section{KEYWORDS}

Soft set;

Three-valued soft set;

Choice value;

Decision making;

TOPSIS;

ELECTRE.

\begin{abstract}
The main objective of the present study is to point to the generalization of Molodtsov's approach to soft sets obtained by passing from the classical two-valued logic underlying those sets to a three-valued logic where the third truth value can usually be interpreted as either non-determined (i.e., between true and false) or unknown. This extension of soft set approach allows a more intuitive and clearer representation of various decision-making problems involving incomplete or uncertain information. In other words, it is a viable approach to analyze soft-set-based multi-criteria-group decision-making problems in the absence of adequate information resulting from the inability to determine the data. In this regard, this study introduced the concept of three-valued soft set and some of its operations and products. In addition, the formulas required to calculate all possible choice values were proposed for each object in the (weighted) three-valued soft sets and their respective decision values were calculated. Both Technique for Order Preference by Similarity to Ideal Solution (TOPSIS) and ELimination Et Choice Translating REality (ELECTRE) methods were modified to deal with multi-criteria group decision problems and then, three-valued soft-set-based decision-making algorithms were constructed. To demonstrate the practicality of these algorithms, the examples adopted from the decision problems in real life were addressed. Lastly, some aspects of the efficiency of the proposed algorithms were discussed using computational experiments.
\end{abstract}

(C) 2021 Sharif University of Technology. All rights reserved.

\section{Introduction}

Classically, a logic is two-valued (Boolean) if every proposition is either false ("0") or true (" 1 "). In 1930, Łukasiewicz [1] initiated a three-valued logic, i.e., a natural extension of the two-valued logic with

*. Corresponding author. Tel.: +90 35424210 21;

Fax: +90 354 242 1022

E-mail addresses: eyup.akcetin@mu.edu.tr (E. Akçetin);

huseyin.kamaci@hotmail.com, huseyin.kamaci@bozok.edu.tr (H. Kamacı)

doi: $10.24200 /$ sci. 2020.54715 .3881 three truth (logical) values indicating false ("0"), true ("1"), and some indeterminate third values (" $\frac{1}{2} "$ as something in the middle between true and false). Further, he pioneered the conceptual form and basic ideas of three-valued logic. By interpreting the intuition of Eukasiewicz three-valued logic from different perspectives, two-valued logic was extended to threevalued logic in different ways [2-5]. Based on their choice of basic connectives, they were different from a syntactic and proof-theoretic point of view. Although the idea of fuzzification of logic was envisaged by several researchers in the years following 1920, the concept of fuzzy logic in which the truth values of 
variables might be any real number between 0 and 1 , both inclusive, was explicitly and crisply proposed by Zadeh [6] in 1965. Fuzzy logic is based on the investigation and observation that people take into account while making decisions based on uncertain and non-numerical information. Fuzzy set, which is a generalization of the crisp set based on the two-valued logic, is the mathematical means of representing vagueness and imprecise information. This set is described by a membership function that assigns to each object a degree of membership ranging between 0 and 1 . Pawlak [7] defined a rough set that can be considered as a new area of uncertainty mathematics closely related to set theory. This set is a formal approximation of a crisp set in a pair of sets which give the lower and upper approximations of the original set. The approximation spaces of rough set theory are sets with multiple memberships, while fuzzy sets are concerned with partial memberships. These sets are combined to derive different variations such as the fuzzy rough set and rough fuzzy set. In 1999, Molodtsov [8] described the soft set based on two-valued logic as a mathematical tool dealing with parametric data which were imprecise or uncertain in nature. In 2003, Maji et al. [9] published a study on the operations of soft sets. Later, the operational laws of the soft sets were derived [1014]. In addition, many authors have described and discussed different types of soft sets such as bijective soft set [15], exclusive disjunctive soft set [16], bipolar soft set [17], inverse soft set $[18,19]$, etc.

Decision making, which is one of the issues that triggers uncertainty, is a frequently encountered problem in many commercial and scientific fields, even in every stage of daily life. For deterministic modeling of decision-making problems, many mathematical techniques such as Technique for Order Preference by Similarity to Ideal Solution (TOPSIS), AHP, VIKOR, ELimination Et Choice Translating (ELECTRE), and PROMETHEE have been developed. These mathematical techniques were adopted for decision-making based on the fuzzy set and some of its extensions [2026]. In addition to the fuzzy modelling of TOPSIS, ELECETRE, etc., many algorithmic solutions were proposed for decision-making in a fuzzy environment [27-31]. In 2002, Maji et al. [32] reported that soft sets could be used to solve decision-making problems involving parametric data based on two-valued logic. In the following years, many soft decision algorithms were created and their applications to problems in real life were specified [33-37]. Moreover, Eraslan [38] proposed a decision-making procedure through the classical method of TOPSIS on the soft sets. In this regard, he pointed out that classical decision-making techniques could be successfully applied to the softset-based decision-making.

In 2008, Avron and Konikowska [39] explored the idea of describing Pawlak's rough set using three-valued logic. This paradigm presents a different perspective in the interpretation of issues involving indeterminate or unknown data in many fields. As with the rough set, three-valued logic emerges in many real-world scenes that are included in the scope of the soft set, and it is difficult to deal with such broadly scoped issues whose third truth value is "undetermined". In this study, a completely designed approach to soft set was discussed using three-valued logic. Thus, in practice, we aim to overcome the difficulties that include a third truth value caused by uncertain or unknown origin, in addition to the truth values of a two-valued logic. The present study was conducted based on the idea of proposing the notion of three-valued soft set to describe a soft set using three-valued logic. The fusion of three-valued logic into the soft set suggests a clearer and intuitive way to explain different issues under incomplete or uncertain information. In this regard, the main focus was put on decision-making based on this type of soft set and offering different algorithmic solutions.

The rest of this paper is organized as follows. Section 2 gives an outline of soft set theory. Section 3 elaborates the motivation to reinterpret soft sets using three-valued logic. Section 4 calculates the choice value of an object in (weighted) three-valued soft sets and accordingly, proposes two algorithms. Section 5 creates a multi-criteria group decision-making algorithm based on the modified TOPSIS on three-valued soft sets. Section 6 proposes a three-valued soft decisionmaking algorithm via the modified ELECTRE, which is based on three fundamental objectives called choosing, sorting, and ranking. In addition, some examples were given to analyze the performance of the algorithms emerging in these two sections. Section 7 solves the matching numerical examples to compare the results of the proposed algorithms, thus showing that they are convincing. The last section presents the concluding remarks and suggests plans for further research.

\section{Preliminaries}

As a preparatory opening for new concepts, this section elaborates some relevant arguments of soft set, twovalued logic, and three-valued logic.

Consider the soft set theory first. In 1999, Molodtsov [8] introduced soft set theory as a useful way of classifying objects based on parametric data. In 2010, Ça g̃man and Enginog̃lu [40] recreated soft sets to make their operations more practical in some cases. Maji et al. [32] put forward that the soft set could be represented in a tabular form. They also demonstrated that soft sets were the parametric sets created based on two-valued logic (i.e., 0 as false, 1 as true). Now, recall the definition of soft set. 
From now on, $\mathfrak{X}$ is a universal set, $\mathcal{P}$ is a parameter set, and $Q \subseteq \mathcal{P}$.

Definition 2.1 $[8,40]$. Assume that $\mathfrak{X}^{\langle\{0,1\}\rangle}$ denotes the set of all functions from $\mathfrak{X}$ to $\{0,1\}$. A pair $\left(f_{Q}, \mathcal{P}\right)=F_{Q}$ is called a soft set over $\mathfrak{X}$ when the mapping $f_{Q}$ is defined by $f_{Q}: \mathcal{P} \rightarrow \mathfrak{X}^{(\{0,1\})}$, where for all $p_{j} \in \mathcal{P}$, the approximate function $f_{Q}\left(p_{j}\right)$ is shown in Box I.

Example 2.1. In daily life, many operations such as shopping, booking, money transfer, etc., can be done through online websites. Recently, some websites have made it feasible to reserve hotel rooms for trips and holidays; take Booking.com and Expedia.com as examples. Now, consider the following problem that one may encounter when making a hotel room reservation.

Let $\mathfrak{X}=\left\{x_{1}, x_{2}, x_{3}, x_{4}, x_{5}\right\}$ be a set of five hotels that are available for booking a room. Let $\mathcal{P}=$ $\left\{p_{1}=\right.$ price, $p_{2}=$ location, $p_{3}=$ amenities, $p_{4}=$ satisfaction $\}$ be a set of the choice parameters. Then, we can create the following soft set over $\mathfrak{X}$ :

$$
\begin{aligned}
T_{\mathcal{P}}= & \left\{\left(p_{1},\left\{x_{1}^{\langle 0\rangle}, x_{2}^{\langle 1\rangle}, x_{3}^{\langle 1\rangle}, x_{4}^{\langle 0\rangle}, x_{5}^{\langle 0\rangle}\right\}\right),\right. \\
& \left(p_{2},\left\{x_{1}^{\langle 1\rangle}, x_{2}^{\langle 1\rangle}, x_{3}^{\langle 0\rangle}, x_{4}^{\langle 0\rangle}, x_{5}^{\langle 1\rangle}\right\}\right), \\
& \left(p_{3},\left\{x_{1}^{\langle 1\rangle}, x_{2}^{\langle 1\rangle}, x_{3}^{\langle 0\rangle}, x_{4}^{\langle 1\rangle}, x_{5}^{\langle 0\rangle}\right\}\right), \\
& \left.\left(p_{4},\left\{x_{1}^{\langle 0\rangle}, x_{2}^{\langle 1\rangle}, x_{3}^{\langle 1\rangle}, x_{4}^{\langle 0\rangle}, x_{5}^{\langle 1\rangle}\right\}\right)\right\} .
\end{aligned}
$$

For the first pair in this soft set, it can be interpreted that the prices of hotels $x_{2}$ and $x_{3}$ are suitable for us, while the prices of hotels $x_{1}, x_{4}$, and $x_{5}$ are not suitable. Other pairs can be interpreted similarly.

In 1930, Eukasiewicz [1] put forward (Lukasiewicz) three-valued logic by extending two-valued logic called Boolean logic. Immediately after the above author, many authors have exhibited their interest in the idea of three-valued logic and its operations $[2,4,39,41,42]$. They argued that since two-valued logic (Boolean logic) could cover all kinds of scientific investigations, three-valued logic might be useful as a basis for a number of useful reasoning tasks. Boolean connectives can be extended to three-valued logic in different ways. In other words, the third truth value can be explained in different ways that are different from true and false. Ciucci and Dubois [43] listed these ways as follows:

- Possible: this explanation was proposed by Łukasiewicz [1] and Borowski [2] the pioneer of three-valued logic. A proposition is regarded as "Possible" if its truth value will be known only in the future;

- Unknown: This explanation was proposed by Kleene [4] in 1952. A proposition is "Unknown" if its truth value cannot be computed for some reasons (for instance, it is too time-consuming to compute);

- Inconsistent: The third value stands for a proposition that is both true and false, and also it is the dual of "Unknown" in some sense;

- Half-true: This is the typical of fuzzy logic [3]. The intuition is that for some propositions, truth is a importance degree. For instance, Shadowed set in $[44,45]$ is based on the idea of turning fuzzy set into three-valued one;

- Undefined: This is another explanation of Kleene [4]. The undefined state corresponds to the selection of the argument of the function outside its definition domain. A proposition is "Undefined" if its truth value involves undefined atoms;

- Irrelevant: The idea behind it is that propositions are not applicable in some possible worlds.

In 1960, Skolem [5] initiated a set theory based on a certain three-valued logic. In this set theory, the variables such as $p, q, r, \ldots$ take three values: $0, \frac{1}{2}, 1$. We may interpret 0 as "false", 1 as "true", and $\frac{1}{2}$ as something in the middle between true and false, say "undetermined". Moreover, Skolem presented a set of truth tables showing tree-valued logic operations like negation, disjunction and conjunction (for a detailed review, see [5]). In the literature, there are many truth tables illustrating tree-valued logic operations. However, this study focused on the truth tables proposed by Skolem [5].

Three-valued logic emerges in several real-world scenes. Three samples in the atmosphere of "undetermined" are presented in Figures 1-3. In these figures, "?" symbolizes "undetermined", i.e., $\frac{1}{2}$.

Since the truth value of "undetermined" in Figures 1 and 2 is precisely known in the future, it can

$$
f_{Q}\left(p_{j}\right)= \begin{cases}\left\{x_{i}^{\left\langle\lambda_{Q_{\left(p_{j}\right)}}^{i}\right\rangle}: x_{i} \in \mathfrak{X} \text { and } \lambda_{f_{Q_{\left(p_{j}\right)}}}^{i} \in\{0,1\}\right\}, & \text { if } p_{j} \in Q \\ \left\{x_{i}^{\langle 0\rangle}: \forall x_{i} \in \mathfrak{X}\right\}, & \text { if } p_{j} \in \mathcal{P}-Q\end{cases}
$$




\begin{tabular}{|c|c|c|c|c|c|c|}
\hline *t & Classify - & Delete & Status & Size & From & Subject \\
\hline \multicolumn{7}{|c|}{$\square$ Good (11 items) } \\
\hline & แ山山 & (1) & soud & $\angle I . Y \mathrm{RD}$ & Assid sallknova (abslaguestelue... & Desl iued: \\
\hline & $9 \sqrt{13}$ & 7 & Good & $7.5 \mathrm{kB}$ & Smart Mobility conference (smar.-. & Smart Mo \\
\hline & $4 \sqrt{19}$ & $\pi$ & Good & $88.3 \mathrm{~KB}$ & Celine (Celine@mta0.sbr08.net) & MWC201: \\
\hline & 4. & 3 & Good & $138.5 \mathrm{~KB}$ & John Morgan (jmorgan@talksate... & talk Satell \\
\hline \multicolumn{7}{|c|}{ — Spam (2 items) } \\
\hline & LIV & $\sqrt{21}$ & Spam & $10.1 \mathrm{~KB}$ & Radio Popular (geral@venca.co... & Hoje pods \\
\hline & $\mathrm{rJ}$ & जा & Spam & $2.6 \mathrm{~KB}$ & BBVA-Seguridad@bbva-secure.es & Cuenta SL \\
\hline \multicolumn{7}{|c|}{ - Undetermined (2 items) } \\
\hline \multirow[t]{2}{*}{ * } & & $\mathbb{3}$ & & $20.0 \mathrm{~KB}$ & Moriana and VanillaPlus (morian... & Many res; \\
\hline & 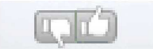 & II & & $8.3 \mathrm{~KB}$ & Hypercel Corp (info@hypercel-m... & New Wick \\
\hline
\end{tabular}

Figure 1. Three-valued logic for classifying mail in a mailbox.

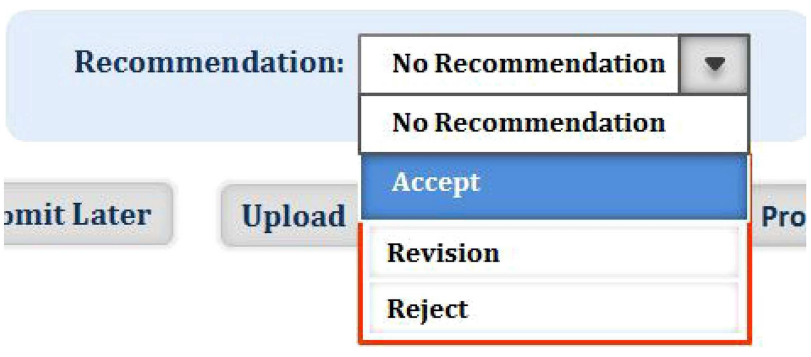

Figure 2. Three-valued logic for reviewers' recommendation in journal.

be considered as "Possible". Figure 3 can also be considered as an example for "Unknown".

\section{Three-valued soft sets}

This section discusses three-valued soft set which is a generalization of soft set obtained by passing from twovalued logic to three-valued one.

The soft set is a set approach proposed by twovalued logic (true and false). In daily life, while evaluating alternatives according to parameters in the decision-making process, "undetermined" mode (neither true nor false, or both true and false) sometimes arises. In such decision-making processes, the soft sets are insufficient. To overcome this shortcoming, the notion of three-valued soft set, which is a soft set based on three-valued logic (i.e., 0 as false, 1 as true, and $\frac{1}{2}$ as "undetermined") is formed.

\subsection{Three-valued soft set}

Definition 3.1. Assume that $\mathfrak{X}\left\langle\left\{0, \frac{1}{2}, 1\right\}\right\rangle$ denotes the set of all functions from $\mathfrak{X}$ to $\left\{0, \frac{1}{2}, 1\right\}$. A pair $\left(t_{Q}, \mathcal{P}\right)=$ $T_{Q}$ is called a three-valued soft set over $\mathfrak{X}$ when the mapping $t_{Q}$ is defined by $t_{Q}: \mathcal{P} \rightarrow \mathfrak{X}^{\left(\left\{0, \frac{1}{2}, 1\right\}\right)}$, where for all $p_{j} \in \mathcal{P}$ the equation shown in Box II is obtained.

Notation: For the parameter set $\mathcal{P}$, the set of all three-valued soft sets over $\mathfrak{X}$ is denoted by $\mathcal{T} \mathcal{S S}(\mathfrak{X}, \mathcal{P})$.

Example 3.1. Assume that $\mathfrak{X}=\left\{x_{1}, x_{2}, x_{3}, x_{4}, x_{5}\right\}$ is a set of drugs that can be taken by pregnant

\begin{tabular}{|c|c|c|c|c|}
\hline Gene Allele Symbol & Rhinosinusitis & Otitis Media & Situs Inversus & Infertifity \\
\hline Ulk4 $4^{\text {tm/Lex }}$ & Yes & Yes & Undetermined & Undetermined \\
\hline Nme5 ${ }^{\text {tm/Lex }}$ & Yes & No & No & Yes \\
\hline$N m e 7^{G t(O S T 31 / 6) L e x}$ & Yes & No & Yes & No \\
\hline Kif27 $7^{\text {Gt(OST41915)Lex }}$ & Yes & Yes & No & Undetermined \\
\hline Stk36 $\sigma^{\text {tm/lex }}$ & No & Yes & No & Yes \\
\hline 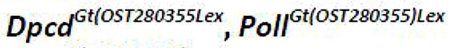 & Yes & No & Yes & Yes \\
\hline$A k 7^{G t(O S T 434404) L e x}$ & Yes & No & No & Yes \\
\hline$A k 8^{\text {Gt(OSTI6378)Lex }}$ & No & No & No & No \\
\hline 4930444A02Rik ${ }^{\text {Gt(OST243203)Lex }}$ & No & No & Undetermined & Yes \\
\hline Celsr2 ${ }^{\text {tmillex }}$ & No & No & No & No \\
\hline Mboat $7^{\text {tm/Lex }}$ & No & No & No & No \\
\hline Tg(FZD3)ILex & No & No & No & No \\
\hline
\end{tabular}

Figure 3. Three-valued logic for cilia-related lesions in hydrocephalic mice (see [46]). 


$$
t_{Q}\left(p_{j}\right)= \begin{cases}\left\{x_{i}^{\left\langle\lambda_{{ }^{2}}^{i}{ }_{Q_{\left(p_{j}\right)}}\right\rangle}: x_{i} \in \mathfrak{X} \text { and } \lambda_{t_{Q_{\left(p_{j}\right)}}^{i}}^{i} \in\left\{0, \frac{1}{2}, 1\right\}\right\}, & \text { if } p_{j} \in Q \\ \left\{x_{i}^{\langle 0\rangle}: \forall x_{i} \in \mathfrak{X}\right\}, & \text { if } p_{j} \in \mathcal{P}-Q\end{cases}
$$

Box II

Table 1. The side effects specified in the prospectus of drugs.

\begin{tabular}{cccc}
\hline & $\boldsymbol{p}_{\mathbf{1}}=$ allergy & $\boldsymbol{p}_{\mathbf{2}}=$ fetal damage & $\boldsymbol{p}_{\mathbf{3}}=$ pharmacological effect \\
\hline$x_{1}$ & $\checkmark$ & $\times$ & - \\
$x_{2}$ & $\times$ & - & $\checkmark$ \\
$x_{3}$ & $\checkmark$ & $\times$ & $\times$ \\
$x_{4}$ & $\times$ & - & - \\
$x_{5}$ & $\times$ & $\checkmark$ & $\times$ \\
\hline
\end{tabular}

Note: The symbols $\checkmark, \times$, and - represent Yes, No and Undetermined, respectively.

women and those of childbearing age. The drugs that can be taken by these women, can be observed without increase in the frequency of malformation or other direct or indirect harmful effects on the human fetus. Therefore, these women should carefully check their side effects while using the drug. Suppose that $\mathcal{P}=\left\{p_{1}=\right.$ allergy, $p_{2}=$ fetal damage, $p_{3}=$ pharmacological effect\} denotes some side effects of the drugs. Here, the following table (Table 1) can be obtained by examining the prospectus of drugs.

Based on Table 1, the following three-valued soft set is constructed:

$$
\begin{aligned}
T_{\mathcal{P}}= & \left\{\left(p_{1},\left\{x_{1}^{\langle 1\rangle}, x_{2}^{\langle 0\rangle}, x_{3}^{\langle 1\rangle}, x_{4}^{\langle 0\rangle}, x_{5}^{\langle 0\rangle}\right\}\right),\right. \\
& \left(p_{2},\left\{x_{1}^{\langle 0\rangle}, x_{2}^{\left\langle\frac{1}{2}\right\rangle}, x_{3}^{\langle 0\rangle}, x_{4}^{\left\langle\frac{1}{2}\right\rangle}, x_{5}^{\langle 1\rangle}\right\}\right), \\
& \left(p_{3},\left\{x_{1}^{\left\langle\frac{1}{2}\right\rangle}, x_{2}^{\langle 1\rangle}, x_{3}^{\langle 0\rangle}, x_{4}^{\left\langle\frac{1}{2}\right\rangle}, x_{5}^{\langle 0\rangle}\right\}\right) .
\end{aligned}
$$

Example 3.2. Let $\mathfrak{X}=\left\{x_{1}=\right.$ Vivo V17 Pro, $x_{2}=$ OnePlus $7, x_{3}=$ Xiaomi Redmi K20 Pro, $x_{4}=$ Apple IPhone 11\} be a set of four mobiles, and
$\mathcal{P}=\left\{p_{1}=F M\right.$ Radio, $p_{2}=$ Stereo Speakers, $p_{3}=$ Loudspeaker $\}$ a set of multimedia features (attributes) that may be available on phones. Through the website "www.91mobiles.com" (date: 17.10.2019), we have Figure 4. Based on this figure, the following threevalued soft set can be created:

$$
\begin{aligned}
T_{\mathcal{P}}= & \left\{\left(p_{1},\left\{x_{1}^{\langle 1\rangle}, x_{2}^{\langle 0\rangle}, x_{3}^{\left\langle\frac{1}{2}\right\rangle}, x_{4}^{\langle 0\rangle}\right\}\right),\right. \\
& \left(p_{2},\left\{x_{1}^{\left\langle\frac{1}{2}\right\rangle}, x_{2}^{\langle 1\rangle}, x_{3}^{\left\langle\frac{1}{2}\right\rangle}, x_{4}^{\langle 1\rangle}\right\}\right), \\
& \left.\left(p_{3},\left\{x_{1}^{\langle 1\rangle}, x_{2}^{\langle 1\rangle}, x_{3}^{\langle 1\rangle}, x_{4}^{\langle 1\rangle}\right\}\right)\right\} .
\end{aligned}
$$

Based on the information presented on this website, we can interpret that for the multimedia feature $p_{1}$ (FM Radio):

- The mobile $x_{1}$ (Vivo V17 Pro) has an FM Radio;

- The mobiles $x_{2}$ and $x_{4}$ (OnePlus 7 and Apple IPhone 11) do not have an FM Radio; and

- It remains "undetermined" whether the mobile $x_{3}$ (Xiaomi Redmi K20 Pro) has an FM Radio.

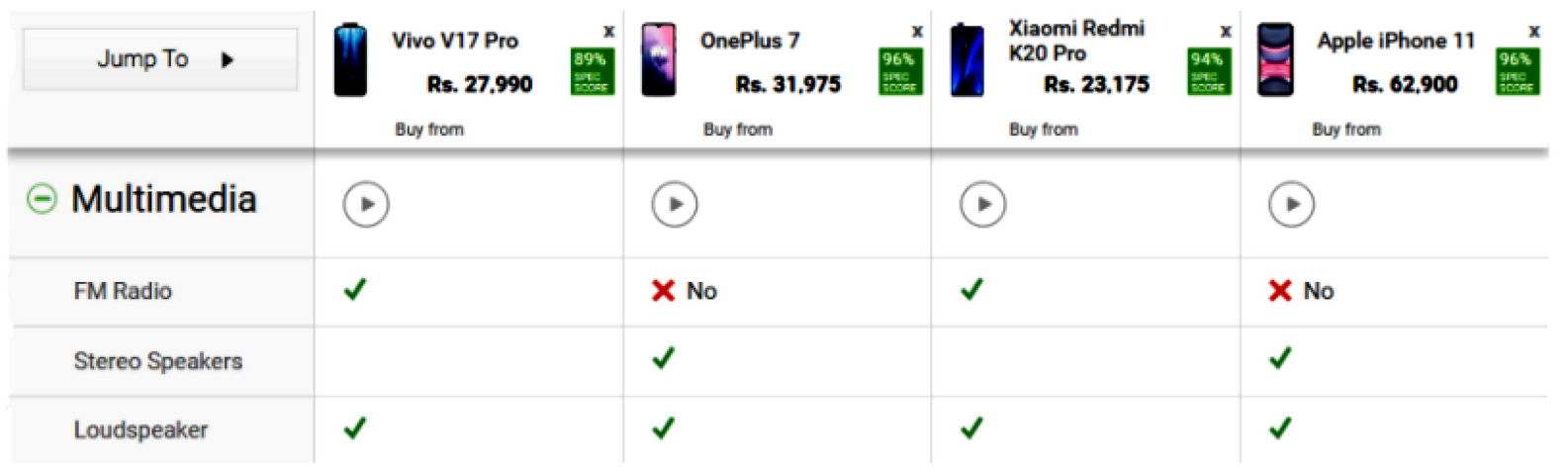

Figure 4. Comparison of some multi-media features of four mobiles. 
Comment: The truth value $\frac{1}{2}$ in the three-valued logic differs from the membership degree 0.5 in the fuzzy logic. The truth value $\frac{1}{2}$ in the three-valued logic represents "undetermined", not membership. Why is this truth value between 0 and 1 , not 0 ? 0 indicates that the given object does not certainly have the desired property, while $\frac{1}{2}$ means that the object might possibly have this property. To be specific, assume that one wants to buy any item from the mobiles $x_{2}$ and $x_{3}$ (presented in Figure 4). As a criterion for purchasing, the requirement to have only FM Radio is determined. In such situations, it is more convenient to select the mobile $x_{3}$. Since it is not known (undetermined) whether or not the mobile $x_{3}$ has an FM Radio and the mobile $x_{2}$ has no FM Radio, there is also the possibility that the mobile $x_{3}$ has an FM Radio. Indeed, we reconsider through the website "www.smartprix.com" that the mobile $x_{3}$ (Xiaomi Redmi K20 Pro) has an FM Radio.

Example 3.3. Let $\mathfrak{X}=\left\{x_{1}, x_{2}, x_{3}, x_{4}\right\}$ be a set of four investments at the disposal of the investor to invest some money and $\mathcal{P}=\left\{p_{1}=\right.$ riskless, $p_{2}=$ security, $p_{3}=$ tax free, $p_{4}=$ short period $\}$ a set of parameters. For parameter subset $Q_{1}=\left\{p_{1}, p_{2}, p_{3}\right\}$, an investor can create the following three-valued soft set over $\mathfrak{X}$ :

$$
\begin{gathered}
T_{Q_{1}}=\left\{\left(p_{1},\left\{x_{1}^{\langle 1\rangle}, x_{2}^{\langle 0\rangle}, x_{3}^{\langle 1\rangle}, x_{4}^{\left\langle\frac{1}{2}\right\rangle}\right\}\right),\right. \\
\left(p_{2},\left\{x_{1}^{\langle 0\rangle}, x_{2}^{\langle 1\rangle}, x_{3}^{\left\langle\frac{1}{2}\right\rangle}, x_{4}^{\langle 1\rangle}\right\}\right), \\
\left.\left(p_{3},\left\{x_{1}^{\langle 1\rangle}, x_{2}^{\left\langle\frac{1}{2}\right\rangle}, x_{3}^{\left\langle\frac{1}{2}\right\rangle}, x_{4}^{\langle 1\rangle}\right\}\right)\right\} .
\end{gathered}
$$

The element $\left(p_{1},\left\{x_{1}^{\langle 1\rangle}, x_{2}^{\langle 0\rangle}, x_{3}^{\langle 1\rangle}, x_{4}^{\left\langle\frac{1}{2}\right\rangle}\right\}\right)$ in $T_{Q_{1}}$ means that:

- The investments $x_{1}$ and $x_{3}$ are risk-free;

- The investment $x_{2}$ is risky; and

- The investment $x_{4}$ is "undetermined" in terms of risk.

As shown in the above example, if $p_{j} \in \mathcal{P} \backslash Q_{1}$, the pair $\left(p_{j}, t_{Q_{1}}\left(p_{j}\right)\right)$ does not need to be displayed in the structure of the three-valued soft set $T_{Q_{1}}$. However, it is known that $\left(p_{j},\left\{x_{i}^{\langle 0\rangle}: \forall x_{i} \in \mathfrak{X}\right\}\right)$.

Each three-valued soft set can be represented in the form of a binary table. This representation makes three-valued soft sets useful in different computer program languages as well as the practicality of calculations.

The binary tabular form of three-valued soft set $T_{Q_{1}}$ in Example 3.3 is presented in Table 2.

In Table 2, each component $x_{i j}$ represents the truth value $\lambda_{t_{Q_{\left(p_{j}\right)}}}^{i}$ of the alternative $x_{i}$ with respect to the parameter $p_{j}$, that is, in Table 2 and onward:
Table 2. The tabular form of three valued soft set $T_{Q_{1}}$ in Example 3.3.

\begin{tabular}{ccccc}
\hline $\mathfrak{X} / \boldsymbol{P}$ & $\boldsymbol{p}_{\boldsymbol{1}}$ & $\boldsymbol{p}_{\boldsymbol{2}}$ & $\boldsymbol{p}_{\mathbf{3}}$ & $\boldsymbol{p}_{\boldsymbol{4}}$ \\
\hline$x_{1}$ & 1 & 0 & 1 & 0 \\
$x_{2}$ & 0 & 1 & $\frac{1}{2}$ & 0 \\
$x_{3}$ & 1 & $\frac{1}{2}$ & $\frac{1}{2}$ & 0 \\
$x_{4}$ & $\frac{1}{2}$ & 1 & 1 & 0 \\
\hline
\end{tabular}

- $x_{i j}=1$ means that $x_{i}$ belongs to the subset of $\mathfrak{X}$ approximated by the parameter $p_{j}$;

- $x_{i j}=0$ means that $x_{i}$ does not belong to the subset of $\mathfrak{X}$ approximated by the parameter $p_{j}$; and

- $x_{i j}=\frac{1}{2}$ means that it is undetermined whether $x_{i}$ belongs to the subset of $\mathfrak{X}$ approximated by the parameter $p_{j}$.

From now on, in the examples, the three-valued soft sets will be represented by the binary tables.

Definition 3.2. Let $T_{Q} \in \mathcal{T} \mathcal{V S S}(\mathfrak{X}, \mathcal{P})$. It is called:

a) An empty three-valued soft set when $t_{Q}\left(p_{j}\right)=$ $\left\{x_{i}^{\langle 0\rangle}: \forall x_{i} \in \mathfrak{X}\right\}$ for all $p_{j} \in \mathcal{P}$ and it is denoted by $T_{\Theta}$

b) A $Q$-mid three-valued soft set when $t_{Q}\left(p_{j}\right)=$ $\left\{x_{i}^{\left\langle\frac{1}{2}\right\rangle}: \forall x_{i} \in \mathfrak{X}\right\}$ for all $p_{j} \in Q$, and it is denoted by $T_{\widetilde{Q}}$. If $Q=\mathcal{P}$, the $Q$-mid three-valued soft set is called a mid three-valued soft set and it is denoted by $T_{\widetilde{\mathcal{P}}}$;

c) A $Q$-universal three-valued soft set when $t_{Q}\left(p_{j}\right)=$ $\left\{x_{i}^{\langle 1\rangle}: \forall x_{i} \in \mathfrak{X}\right\}$ for all $p_{j} \in Q$, and it is denoted by $T_{\widehat{Q}}$. If $Q=\mathcal{P}$, the $Q$-universal three-valued soft set is called a universal three-valued soft set, and it is denoted by $T_{\widehat{\mathcal{P}}}$.

Definition 3.3. Let $T_{Q_{1}}, T_{Q_{2}} \in \mathcal{T} \mathcal{V S S}(\mathfrak{X}, \mathcal{P})$, then, we have:

a) $T_{Q_{1}}$ is a three-valued soft subset of $T_{Q_{2}}$ when $t_{Q_{1}}\left(p_{j}\right) \subseteq t_{Q_{2}}\left(p_{j}\right)$ for all $p_{j} \in P$ and it is denoted by $T_{Q_{1}} \sqsubseteq T_{Q_{2}}$. Here, $t_{Q_{1}}\left(p_{j}\right) \subseteq t_{Q_{2}}\left(p_{j}\right)$ for $p_{j} \in \mathcal{P}$ means $\lambda_{t_{Q_{1}\left(p_{j}\right)}}^{i} \leq \lambda_{t_{Q_{2}\left(p_{j}\right)}}^{i}$ for each $x_{i} \in \mathfrak{X}$.

b) $T_{Q_{1}}$ and $T_{Q_{2}}$ are both equal three-valued soft sets when $t_{Q_{1}}\left(p_{j}\right)=t_{Q_{2}}\left(p_{j}\right)$ for all $p_{j} \in P$, denoted by $T_{Q_{1}}=T_{Q_{2}}$. Here, $t_{Q_{1}}\left(p_{j}\right)=t_{Q_{2}}\left(p_{j}\right)$ for $p_{j} \in \mathcal{P}$ means that $\lambda_{t_{Q_{1}\left(p_{j}\right)}}^{i}=\lambda_{t_{Q_{2}\left(p_{j}\right)}}^{i}$ for each $x_{i} \in \mathfrak{X}$.

Example 3.4. Let us consider the three-valued soft set $T_{Q_{1}}$ given in Table 2 of Example 3.3. In addition, the three-valued soft set $T_{Q_{2}}$ is shown in Table 3 . Then, $T_{Q_{2}} \sqsubseteq T_{Q_{1}}$.

Proposition 3.1. Let $T_{Q_{1}}, T_{Q_{2}}, T_{Q_{3}} \in \mathcal{T} \mathcal{V S S}(\mathfrak{X}, \mathcal{P})$. 
Table 3. The tabular form of $T_{Q_{2}}$ for $Q_{2}=\mathcal{P}$.

\begin{tabular}{ccccc}
\hline $\mathfrak{X} / \mathcal{P}$ & $\boldsymbol{p}_{\mathbf{1}}$ & $\boldsymbol{p}_{\mathbf{2}}$ & $\boldsymbol{p}_{\mathbf{3}}$ & $\boldsymbol{p}_{\boldsymbol{4}}$ \\
\hline$x_{1}$ & $\frac{1}{2}$ & 0 & $\frac{1}{2}$ & 0 \\
$x_{2}$ & 0 & 1 & $\frac{1}{2}$ & 0 \\
$x_{3}$ & $\frac{1}{2}$ & 0 & 0 & 0 \\
$x_{4}$ & 0 & 1 & 1 & 0 \\
\hline
\end{tabular}

i. $T_{\Theta} \sqsubseteq T_{\widetilde{\mathcal{P}}} \sqsubseteq T_{\widehat{P}} ;$

ii. $T_{\Theta} \sqsubseteq T_{Q_{k}}$ for each $k$;

iii. $T_{Q_{k}} \sqsubseteq T_{\widehat{p}}$;

iv. $T_{Q_{k}} \sqsubseteq T_{Q_{k}}$ for each $k$;

v. $T_{Q_{1}} \sqsubseteq T_{Q_{2}}$ and $T_{Q_{2}} \sqsubseteq T_{Q_{3}} \Rightarrow T_{Q_{1}} \sqsubseteq T_{Q_{3}}$.

Proof. The proofs are obvious according to Definitions 3.2 and 3.3 , hence they are omitted. $\square$

\subsection{Operations and products on three-valued soft sets}

Definition 3.4. Let $T_{Q} \in \mathcal{T} \mathcal{V S S}(\mathfrak{X}, \mathcal{P})$. Then the complement of $T_{Q}$, denoted by $\overline{T_{Q}}$, is defined by the mapping $\overline{t_{Q}}: \mathcal{P} \rightarrow \mathfrak{X}^{\left(\left\{0, \frac{1}{2}, 1\right\}\right)}$ such that:

$$
\begin{aligned}
\overline{t_{Q}}\left(p_{j}\right)= & \left\{x_{i}^{\left\langle\lambda_{\text {tinet } Q_{\left(p_{j}\right)}}^{i}\right\rangle}: x_{i} \in \mathfrak{X}\right. \text { and } \\
& \left.\lambda \bar{t}_{\bar{Q}_{\left(p_{j}\right)}}^{i} \in\left\{0, \frac{1}{2}, 1\right\}\right\},
\end{aligned}
$$

for all $p_{j} \in \mathcal{P}$ where:

$$
\lambda_{\bar{t}_{\left(p_{j}\right)}}^{i}=1-\lambda_{t_{Q_{\left(p_{j}\right)}}^{i}}
$$

Note: This definition clarifies why the truth value for "undetermined" is $\frac{1}{2}$. The negation of "undetermined" must also have the same truth value because there is no gauge of "undetermined". Accordingly, we argue
Table 4. The tabular form of $T_{Q_{2}}$ for $Q_{2}=\mathcal{P}$.

\begin{tabular}{ccccc}
\hline $\mathfrak{X} / \mathcal{P}$ & $\boldsymbol{p}_{\mathbf{1}}$ & $\boldsymbol{p}_{\mathbf{2}}$ & $\boldsymbol{p}_{\boldsymbol{3}}$ & $\boldsymbol{p}_{\mathbf{4}}$ \\
\hline$x_{1}$ & $\frac{1}{2}$ & 1 & 1 & 0 \\
$x_{2}$ & 0 & 0 & 0 & 0 \\
$x_{3}$ & $\frac{1}{2}$ & $\frac{1}{2}$ & 1 & 0 \\
$x_{4}$ & 1 & 0 & 0 & 1 \\
\hline
\end{tabular}

Table 5. The tabular form of $\overline{T_{Q_{2}}}$.

\begin{tabular}{ccccc}
\hline $\mathfrak{X} / \mathcal{P}$ & $\boldsymbol{p}_{\mathbf{1}}$ & $\boldsymbol{p}_{\mathbf{2}}$ & $\boldsymbol{p}_{\mathbf{3}}$ & $\boldsymbol{p}_{\mathbf{4}}$ \\
\hline$x_{1}$ & $\frac{1}{2}$ & 0 & 0 & 1 \\
$x_{2}$ & 1 & 1 & 1 & 1 \\
$x_{3}$ & $\frac{1}{2}$ & $\frac{1}{2}$ & 0 & 1 \\
$x_{4}$ & 0 & 1 & 1 & 0 \\
\hline
\end{tabular}

that "undetermined" implies something in the middle between true and false, whose truth vale is $\frac{1}{2}$.

Example 3.5. We consider the universal set $\mathfrak{X}$ and parameter set $\mathcal{P}$ in Example 3.3. Furthermore, we generated the three-valued soft set $T_{Q_{2}}$ given in Table 4 .

The complement of $T_{Q_{2}}$ is obtained and shown in Table 5.

Proposition 3.2. Let $T_{Q} \in \mathcal{T} \mathcal{V S S}(\mathfrak{X}, \mathcal{P})$.

i. $\overline{\left(\overline{T_{Q}}\right)}=T_{Q}$;

ii. $\overline{T_{\Theta}}=T_{\widehat{p}}$;

iii. $\overline{T_{\widetilde{\mathcal{P}}}}=T_{\widetilde{\mathcal{P}}}$.

Proof. (i) We consider three-valued soft set $T_{Q}=$ $\left(t_{Q}, \mathcal{P}\right)$ over $\mathfrak{X}$. Then, we have the mapping $t_{Q}: \mathcal{P} \rightarrow$ $\mathfrak{X}^{\left(\left\{0, \frac{1}{2}, 1\right\}\right)}$ so that for all $p_{j} \in \mathcal{P}$ we obtain the equation shown in Box III. Based on Definition 3.4, we can write for all $p_{j} \in \mathcal{P}$ the equation shown in Box IV. When proceeding in a similar manner, for all $p_{j} \in \mathcal{P}$ we

$$
t_{Q}\left(p_{j}\right)= \begin{cases}\left\{x_{i}^{\left\langle\lambda_{t_{\left(p_{j}\right)}^{i}}^{i}\right\rangle}: x_{i} \in \mathfrak{X} \text { and } \lambda_{t_{\left(p_{j}\right)}}^{i} \in\left\{0, \frac{1}{2}, 1\right\}\right\}, & \text { if } p_{j} \in Q \\ \left\{x_{i}^{\langle 0\rangle}: \forall x_{i} \in \mathfrak{X}\right\}, & \text { if } p_{j} \in \mathcal{P}-Q\end{cases}
$$

$$
\overline{t_{Q}}\left(p_{j}\right)= \begin{cases}\left\{x_{i}^{\left\langle 1-\lambda_{t_{Q_{\left(p_{j}\right)}}^{i}}\right\rangle}: x_{i} \in \mathfrak{X} \text { and } \lambda_{t_{Q_{\left(p_{j}\right)}}}^{i} \in\left\{0, \frac{1}{2}, 1\right\}\right\}, & \text { if } p_{j} \in Q \\ \left\{x_{i}^{\langle 1-0\rangle}: \forall x_{i} \in \mathfrak{X}\right\}, & \text { if } p_{j} \in \mathcal{P}-Q\end{cases}
$$




$$
\begin{aligned}
\overline{\overline{t_{Q}}}\left(p_{j}\right)= \begin{cases}\left\{x_{i}^{\left\langle 1-\left(1-\lambda_{Q_{Q_{\left(p_{j}\right)}}^{i}}\right)\right\rangle}: x_{i} \in \mathfrak{X} \text { and } \lambda_{t_{Q_{\left(p_{j}\right)}}^{i}}^{i} \in\left\{0, \frac{1}{2}, 1\right\}\right\}, & \text { if } p_{j} \in Q \\
\left\{x_{i}^{\langle 1-(1-0)\rangle}: \forall x_{i} \in \mathfrak{X}\right\}, & \text { if } p_{j} \in \mathcal{P}-Q\end{cases} \\
= \begin{cases}\left\{x_{i}^{\left\langle\lambda_{t_{\left(p_{j}\right)}^{i}}^{i}\right\rangle}: x_{i} \in \mathfrak{X} \text { and } \lambda_{t_{Q_{\left(p_{j}\right)}}^{i}}^{i} \in\left\{0, \frac{1}{2}, 1\right\}\right\}, & \text { if } p_{j} \in Q \\
\left\{x_{i}^{\langle 0\rangle}: \forall x_{i} \in \mathfrak{X}\right\}, & \text { if } p_{j} \in \mathcal{P}-Q\end{cases}
\end{aligned}
$$

\section{Box V}

obtained the equation shown in Box V. Therefore, we have $\overline{\overline{t_{Q}}}\left(p_{j}\right)=t_{Q}\left(p_{j}\right)$ for all $p_{j} \in \mathcal{P}$. So, $\overline{\left(\overline{T_{Q}}\right)}=T_{Q}$. omitted.

Proofs (ii) and (iii) are obvious, hence they are

Definition 3.5. Let $T_{Q_{1}}, T_{Q_{2}} \in \mathcal{T} \mathcal{V S S}(\mathfrak{X}, \mathcal{P})$. Then, the intersection of $T_{Q_{1}}$ and $T_{Q_{2}}$, denoted by $T_{Q_{1}} \bar{\Pi} T_{Q_{2}}$, is defined by the mapping $t_{Q_{1} \bar{\Pi} Q_{2}}: \mathcal{P} \rightarrow \mathfrak{X}^{\left(\left\{0, \frac{1}{2}, 1\right\}\right)}$ such that:

$$
\begin{aligned}
t_{Q_{1} \bar{\Pi} Q_{2}}\left(p_{j}\right)= & \left\{x_{i}^{\left\langle\lambda_{t\left(Q_{1} \bar{\Pi} Q_{2}\right)_{\left(p_{j}\right)}}^{i}\right\rangle}: x_{i} \in \mathfrak{X}\right. \text { and } \\
& \left.\lambda_{t_{\left(Q_{1} \sqcap Q_{2}\right)_{\left(p_{j}\right)}}^{i}}^{i} \in\left\{0, \frac{1}{2}, 1\right\}\right\},
\end{aligned}
$$

for all $p_{j} \in \mathcal{P}$, where:

$$
\lambda_{t_{\left(Q_{1} \sqcap Q_{2}\right)\left(p_{j}\right)}}^{i}=\min \left\{\lambda_{t_{Q_{1\left(p_{j}\right)}}^{i}}^{i}, \lambda_{t_{Q_{2\left(p_{j}\right)}}}^{i}\right\} .
$$

Proposition 3.3. Let $T_{Q} \in \mathcal{T} \mathcal{V S S}(\mathfrak{X}, \mathcal{P})$.
i. $T_{Q} \bar{\Pi} T_{\Theta}=T_{\Theta}$;
ii. $T_{Q} \bar{\Pi} T_{\widehat{Q}}=T_{Q}$
iii. $T_{Q} \bar{\Pi} T_{\widetilde{Q}} \sqsubseteq T_{Q}$.

Proof. It is clear from Definitions 3.2, 3.3, and 3.5.

Definition 3.6. Let $T_{Q_{1}}, T_{Q_{2}} \in \mathcal{T} \mathcal{V S S}(\mathfrak{X}, \mathcal{P})$. Then, the union of $T_{Q_{1}}$ and $T_{Q_{2}}$, denoted by $T_{Q_{1}} \sqcup T_{Q_{2}}$, is defined by the mapping $t_{Q_{1} \unlhd Q_{2}}: \mathcal{P} \rightarrow \mathfrak{X}^{\left(\left\{0, \frac{1}{2}, 1\right\}\right)}$ such that:

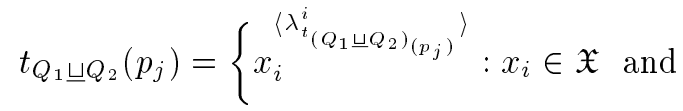

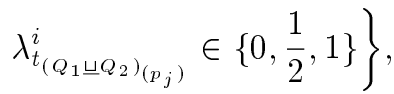

for all $p_{j} \in \mathcal{P}$, where:

$$
\lambda_{t_{\left(Q_{1} \unrhd Q_{2}\right)\left(p_{j}\right)}}^{i}=\max \left\{\lambda_{t_{Q_{1\left(p_{j}\right)}}}^{i}, \lambda_{t_{Q_{2\left(p_{j}\right)}}}^{i}\right\} \text {. }
$$

Proposition 3.4. Let $T_{Q} \in \mathcal{T} \mathcal{V S S}(\mathfrak{X}, \mathcal{P})$. i. $T_{Q} \sqsubseteq T_{\Theta}=T_{Q}$;

ii. $T_{Q} \sqsubseteq T_{\widehat{Q}}=T_{\widehat{Q}}$;

iii. $T_{Q} \sqsubseteq T_{\widetilde{Q}} \sqsupseteq T_{Q}$.

Proof. It is clear from Definitions 3.2, 3.3, and 3.6.

Proposition 3.5. Let $T_{Q_{1}}, T_{Q_{2}}, T_{Q_{3}} \in \mathcal{T} \mathcal{V S S}(\mathfrak{X}, \mathcal{P})$. For all $*, \circ \in\{\bar{\Pi}, \underline{\sqcup}\}$ :
i. $T_{Q_{k}} * T_{Q_{k}}=T_{Q_{k}}$;
ii. $T_{Q_{1}} *\left(T_{Q_{2}} * T_{Q_{3}}\right)=\left(T_{Q_{1}} * T_{Q_{2}}\right) * T_{Q_{3}}$;
iii. $T_{Q_{1}} *\left(T_{Q_{2}} \circ T_{Q_{3}}\right)=\left(T_{Q_{1}} * T_{Q_{2}}\right) \circ\left(T_{Q_{1}} * T_{Q_{3}}\right)$.

Proof. Proofs (i) and (ii) are similar to (iii), hence omitted. (iii) Let us prove that $T_{Q_{1}} *\left(T_{Q_{2}} \circ T_{Q_{3}}\right)=$ $\left(T_{Q_{1}} * T_{Q_{2}}\right) \circ\left(T_{Q_{1}} * T_{Q_{3}}\right)$ for $*=\bar{\Pi}$ and $\circ=\underline{~}$.

We consider $T_{Q_{1}} \bar{\Pi}\left(T_{Q_{2}} \sqcup T_{Q_{3}}\right)$. Suppose that $T_{Q_{2}} \underline{ } T_{Q_{3}}=T_{R}$, where for all $p_{j} \in \mathcal{P}$ :

$$
\begin{gathered}
t_{R}\left(p_{j}\right)=t_{Q_{2} \unlhd Q_{3}}\left(p_{j}\right)=\left\{x_{i}^{\left\langle\lambda_{t_{R}\left(p_{j}\right)}^{i}\right\rangle}: x_{i} \in \mathfrak{X}\right. \text { and } \\
\left.\lambda_{t_{R}\left(p_{j}\right)}^{i}=\lambda_{t_{\left(Q_{2} \unrhd Q_{3}\right)_{\left(p_{j}\right)}}^{i}}^{i} \in\left\{0, \frac{1}{2}, 1\right\}\right\},
\end{gathered}
$$

such that:

$$
\lambda_{t_{R_{\left(p_{j}\right)}}}^{i}=\lambda_{t_{\left(Q_{2} \underline{\Perp} Q_{3}\right)_{\left(p_{j}\right)}}^{i}}^{i}=\max \left\{\lambda_{t_{Q_{2\left(p_{j}\right)}}}^{i}, \lambda_{t_{Q_{3\left(p_{j}\right)}}}^{i}\right\} \text {. }
$$

Assume that $T_{Q_{1}} \Pi T_{R}=T_{S}$, where for all $p_{j} \in \mathcal{P}$ :

$$
\begin{gathered}
t_{S}\left(p_{j}\right)=t_{Q_{1} \sqcap R}\left(p_{j}\right)=\left\{x_{i}^{\left\langle\lambda_{t_{S}\left(p_{j}\right)}^{i}\right\rangle}: x_{i} \in \mathfrak{X}\right. \text { and } \\
\left.\lambda_{t_{S}\left(p_{j}\right)}^{i}=\lambda_{t_{\left(Q_{1} \sqcap R\right)_{\left(p_{j}\right)}}^{i}}^{i} \in\left\{0, \frac{1}{2}, 1\right\}\right\},
\end{gathered}
$$

such that:

$$
\begin{aligned}
\lambda_{t_{S_{\left(p_{j}\right)}}}^{i} & =\lambda_{t_{\left(Q_{1} \Pi R\right)_{\left(p_{j}\right)}}}^{i}=\min \left\{\lambda_{t_{Q_{1\left(p_{j}\right)}}}^{i}, \lambda_{t_{R_{\left(p_{j}\right)}}}^{i}\right\} \\
& =\min \left\{\lambda_{t_{Q_{Q_{\left(p_{j}\right)}}}^{i}}^{i}, \max \left\{\lambda_{t_{Q_{Q_{\left(p_{j}\right)}}}^{i}}^{i}, \lambda_{t_{Q_{3\left(p_{j}\right)}}}^{i}\right\}\right\} .
\end{aligned}
$$


Now, we consider $\left(T_{Q_{1}} \bar{\Pi} T_{Q_{2}}\right) \sqcup\left(T_{Q_{1}} \bar{\Pi} T_{Q_{3}}\right)$. Assume that $T_{Q_{1}} \sqcap T_{Q_{2}}=T_{U}$, where for all $p_{j} \in \mathcal{P}$ :

$$
\begin{gathered}
t_{U}\left(p_{j}\right)=t_{Q_{1} \bar{\Pi} Q_{2}}\left(p_{j}\right)=\left\{x_{i}^{\left\langle\lambda_{t_{U}\left(p_{j}\right)}^{i}\right\rangle}: x_{i} \in \mathfrak{X}\right. \text { and } \\
\left.\lambda_{t_{U}\left(p_{j}\right)}^{i}=\lambda_{t_{\left(Q_{1} \Pi Q_{2}\right)_{\left(p_{j}\right)}}^{i}}^{i} \in\left\{0, \frac{1}{2}, 1\right\}\right\},
\end{gathered}
$$

such that:

$$
\lambda_{t_{U_{\left(p_{j}\right)}}}^{i}=\lambda_{t_{\left(Q_{1} \sqcap Q_{2}\right)_{\left(p_{j}\right)}}^{i}}^{i}=\min \left\{\lambda_{t_{Q_{1\left(p_{j}\right)}}}^{i}, \lambda_{t_{Q_{2\left(p_{j}\right)}}}^{i}\right\} .
$$

Assume that $T_{Q_{1}} \sqcap T_{Q_{3}}=T_{V}$, where for all $p_{j} \in \mathcal{P}$ :

$$
\begin{gathered}
t_{V}\left(p_{j}\right)=t_{Q_{1} \Pi Q_{2}}\left(p_{j}\right)=\left\{x_{i}^{\left\langle\lambda_{t_{V}\left(p_{j}\right)}^{i}\right\rangle}: x_{i} \in \mathfrak{X}\right. \text { and } \\
\left.\lambda_{t_{V}\left(p_{j}\right)}^{i}=\lambda_{t_{\left(Q_{1} \sqcap Q_{2}\right)_{\left(p_{j}\right)}}^{i}}^{i} \in\left\{0, \frac{1}{2}, 1\right\}\right\},
\end{gathered}
$$

such that:

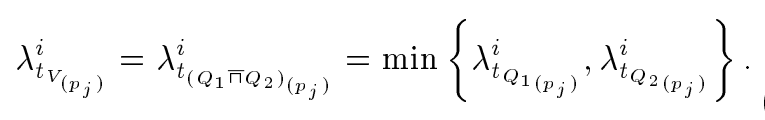

Suppose that $T_{U} \succeq T_{V}=T_{W}$, where for all $p_{j} \in \mathcal{P}$ :

$$
\begin{gathered}
t_{W}\left(p_{j}\right)=t_{U \unlhd V}\left(p_{j}\right)=\left\{x_{i}^{\left\langle\lambda_{t_{W}\left(p_{j}\right)}^{i}\right\rangle}: x_{i} \in \mathfrak{X}\right. \text { and } \\
\left.\lambda_{t_{W}\left(p_{j}\right)}^{i}=\lambda_{t_{(U \unrhd V)_{\left(p_{j}\right)}}^{i}}^{i} \in\left\{0, \frac{1}{2}, 1\right\}\right\},
\end{gathered}
$$

such that:

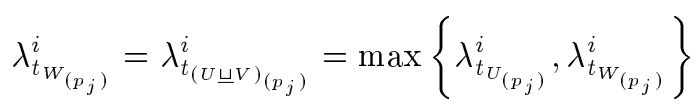

$$
\begin{aligned}
& =\max \left\{\min \left\{\lambda_{t_{Q_{1\left(p_{j}\right)}}}^{i}, \lambda_{t_{Q_{2\left(p_{j}\right)}}}^{i}\right\}\right. \\
& \left.\min \left\{\lambda_{t_{Q_{1\left(p_{j}\right)}}}^{i}, \lambda_{t_{Q_{3\left(p_{j}\right)}}}^{i}\right\}\right\}
\end{aligned}
$$

Since $\lambda_{t_{Q_{1\left(p_{j}\right)}}}^{i}, \lambda_{t_{Q_{2\left(p_{j}\right)}}}^{i}, \lambda_{t_{Q_{3\left(p_{j}\right)}}}^{i} \in\left\{0, \frac{1}{2}, 1\right\}$, we have:

$$
\lambda_{t_{S_{\left(p_{j}\right)}}}^{i}=\lambda_{t_{W_{\left(p_{j}\right)}}^{i}}^{i}
$$

for all $p_{j} \in \mathcal{P}$ (by Eqs. (5) and (8). Therefore, it can be concluded that $T_{S}$ and $T_{W}$ are indeed the same set-valued mappings, and $T_{Q_{1}} \bar{\Pi}\left(T_{Q_{2}} \sqcup T_{Q_{3}}\right)=$ $\left(T_{Q_{1}} \bar{\Pi} T_{Q_{2}}\right) \sqsubseteq\left(T_{Q_{1}} \bar{\Pi} T_{Q_{3}}\right)$.

Other cases can be proved in a similar way.

Proposition 3.6. Let $T_{Q_{1}}, T_{Q_{2}} \in \mathcal{T} \mathcal{V S S}(\mathfrak{X}, \mathcal{P})$. Then, the following De Morgan's rules are held: i. $\overline{\left(T_{Q_{1}} \bar{\Pi} T_{Q_{2}}\right)}=\overline{T_{Q_{1}}} \sqcup \overline{T_{Q_{2}}}$;

ii. $\overline{\left(T_{Q_{1}} \sqcup T_{Q_{2}}\right)}=\overline{T_{Q_{1}}} \sqcap \overline{T_{Q_{2}}}$.

Proof. (i) Since $1-\min \left\{\lambda_{t_{Q_{1}\left(p_{j}\right)}}^{i}, \lambda_{t_{Q_{2}\left(p_{j}\right)}}^{i}\right\}=\max \{1-$ $\left.\lambda_{t_{Q_{1}\left(p_{j}\right)}}^{i}, 1-\lambda_{t_{Q_{2}\left(p_{j}\right)}}^{i}\right\}$ for $\lambda_{t_{Q_{1}\left(p_{j}\right)}}^{i}, \lambda_{t_{Q_{2}\left(p_{j}\right)}}^{i} \in\left\{0, \frac{1}{2}, 1\right\}$, we can say that $\overline{\left(T_{Q_{1}} \bar{\Pi} T_{Q_{2}}\right)}=\overline{T_{Q_{1}}} \sqcup \overline{T_{Q_{2}}}$. (ii) It is similar to the proof of (i).

Definition 3.7. Let $T_{Q_{1}}, T_{Q_{2}} \in \mathcal{T} \mathcal{V S S}(\mathfrak{X}, \mathcal{P})$. Then, the difference between $T_{Q_{1}}$ and $T_{Q_{2}}$, denoted by $T_{Q_{1}} \backslash T_{Q_{2}}$, is defined by the mapping $t_{Q_{1} \backslash Q_{2}}: \mathcal{P} \rightarrow$ $\mathfrak{X}^{\left(\left\{0, \frac{1}{2}, 1\right\}\right)}$ such that:

$$
\begin{aligned}
& t_{Q_{1} \backslash Q_{2}}\left(p_{j}\right)=\left\{x^{\left\langle\lambda_{t}^{i}\left(Q_{1}\left\lfloor Q_{2}\right)_{\left(p_{j}\right)}\right\rangle\right.}: x_{i} \in \mathfrak{X}\right. \text { and } \\
& \left.\lambda_{t_{\left(Q_{1} \backslash Q_{2}\right)\left(p_{j}\right)}}^{i} \in\left\{0, \frac{1}{2}, 1\right\}\right\},
\end{aligned}
$$

for all $p_{j} \in \mathcal{P}$, where:

$$
\lambda_{t_{\left(Q_{1} \underline{Q}_{2}\right)_{\left(p_{j}\right)}}^{i}}^{i}=\min \left\{\lambda_{t_{Q_{1\left(p_{j}\right)}}^{i}}^{i}, 1-\lambda_{t_{Q_{2}\left(p_{j}\right)}}^{i}\right\} .
$$

Definition 3.8. Let $T_{Q_{1}}, T_{Q_{2}} \in \mathcal{T} \mathcal{V S S}(\mathfrak{X}, \mathcal{P})$. Then, the symmetric difference between $T_{Q_{1}}$ and $T_{Q_{2}}$, denoted by $T_{Q_{1}} \triangle T_{Q_{2}}$, is defined by the mapping $t_{Q_{1}} \triangle Q_{2}$ : $\mathcal{P} \rightarrow \mathfrak{X}^{\left(\left\{0, \frac{1}{2}, 1\right\}\right)}$ such that:

$$
\begin{aligned}
t_{Q_{1} \unlhd Q_{2}}\left(p_{j}\right)= & \left\{x_{i}^{\left\langle\lambda_{t}^{i}\left(Q_{1} \triangleq Q_{2}\right)_{\left(p_{j}\right)}\right\rangle}: x_{i} \in \mathfrak{X}\right. \text { and } \\
& \left.\lambda_{t_{\left(Q_{1} \triangleq Q_{2}\right)_{\left(p_{j}\right)}}^{i}}^{i} \in\left\{0, \frac{1}{2}, 1\right\}\right\}
\end{aligned}
$$

for all $p_{j} \in \mathcal{P}$, where:

$$
\begin{aligned}
& \lambda_{t_{\left(Q_{1} \triangle Q_{2}\right)_{\left(p_{j}\right)}}^{i}}^{i}=\min \left\{\max \left\{\lambda_{t_{Q_{1}\left(p_{j}\right)}}^{i}, \lambda_{t_{Q_{2}\left(p_{j}\right)}}^{i}\right\},\right. \\
& \left.1-\min \left\{\lambda_{t_{Q_{1\left(p_{j}\right)}}^{i}}^{i}, \lambda_{t_{Q_{2}\left(p_{j}\right)}}^{i}\right\}\right\}
\end{aligned}
$$

Example 3.6. Consider three-valued soft sets $T_{Q_{1}}$ and $T_{Q_{2}}$ in Tables 2 and 4 , respectively. Therefore, the difference and symmetric difference between $T_{Q_{1}}$ and $T_{Q_{2}}$ can be measured as shown in Tables 6 and 7 , respectively.

Proposition 3.7. Let $T_{Q_{1}}, T_{Q_{2}} \in \mathcal{T} \mathcal{V S S}(\mathfrak{X}, \mathcal{P})$.

i. $T_{Q_{1}} \backslash T_{Q_{2}}=T_{Q_{1}} \bar{\sqcap} \overline{T_{Q_{2}}}$;

ii. $T_{Q_{1}} \triangle T_{Q_{2}}=\left(T_{Q_{1}} \backslash T_{Q_{2}}\right) \sqsubseteq\left(T_{Q_{2}} \backslash T_{Q_{1}}\right)$.

\section{Proof.}


Table 6. The tabular form of $T_{Q_{1}} \backslash T_{Q_{2}}$.

\begin{tabular}{ccccc}
\hline $\mathfrak{X} / \mathcal{P}$ & $\boldsymbol{p}_{\mathbf{1}}$ & $\boldsymbol{p}_{\mathbf{2}}$ & $\boldsymbol{p}_{\boldsymbol{3}}$ & $\boldsymbol{p}_{\mathbf{4}}$ \\
\hline$x_{1}$ & $\frac{1}{2}$ & 0 & 0 & 0 \\
$x_{2}$ & 0 & 1 & $\frac{1}{2}$ & 0 \\
$x_{3}$ & $\frac{1}{2}$ & $\frac{1}{2}$ & 0 & 0 \\
$x_{4}$ & 0 & 1 & 1 & 0 \\
\hline
\end{tabular}

Table 7. The tabular form of $T_{Q_{1}} \triangle T_{Q_{2}}$.

\begin{tabular}{ccccc}
\hline $\mathfrak{X} / \mathcal{P}$ & $\boldsymbol{p}_{\mathbf{1}}$ & $\boldsymbol{p}_{\mathbf{2}}$ & $\boldsymbol{p}_{\mathbf{3}}$ & $\boldsymbol{p}_{\mathbf{4}}$ \\
\hline$x_{1}$ & $\frac{1}{2}$ & 1 & 0 & 0 \\
$x_{2}$ & 0 & 1 & $\frac{1}{2}$ & 0 \\
$x_{3}$ & $\frac{1}{2}$ & $\frac{1}{2}$ & $\frac{1}{2}$ & 0 \\
$x_{4}$ & $\frac{1}{2}$ & 1 & 1 & 1 \\
\hline
\end{tabular}

(i) Assume that $T_{Q_{1}} \backslash T_{Q_{2}}=T_{R}$. According to Eq. (9), for all $p_{j} \bar{\in} \mathcal{P}$, we have:

$$
\lambda_{t_{R}}^{i}\left(p_{j}\right)=\min \left\{\lambda_{t_{Q_{1\left(p_{j}\right)}}^{i}}^{i}, 1-\lambda_{t_{Q_{2}\left(p_{j}\right)}}^{i}\right\} .
$$

On the contrary, assume that $T_{Q_{1}} \overline{\Pi T_{Q_{2}}}=T_{S}$. Then, based on Definition 3.5, we have:

$$
\begin{aligned}
\lambda_{t_{S}}^{i}\left(p_{j}\right)= & \min \left\{\lambda_{t_{Q_{1}\left(p_{j}\right)}}^{i}, \lambda_{t_{Q_{2}\left(p_{j}\right)}}^{i}\right\}= \\
& \min \left\{\lambda_{t_{Q_{\left.1_{(} p_{j}\right)}}}^{i}, 1-\lambda_{t_{Q_{2}\left(p_{j}\right)}}^{i}\right\} .
\end{aligned}
$$

By Eqs. (11) and (12), we prove that the above equality is achieved.

(ii) Assume $T_{Q_{1}} \triangle T_{Q_{2}}=T_{U}$. Then, by Eq. (10), we have for all $\overline{p_{j}} \in \mathcal{P}$ :

$$
\begin{aligned}
\lambda_{t_{U}}^{i}\left(p_{j}\right)= & \min \left\{\max \left\{\lambda_{t_{Q_{1\left(p_{j}\right)}}}^{i}, \lambda_{t_{Q_{2\left(p_{j}\right)}}}^{i}\right\},\right. \\
& \left.1-\min \left\{\lambda_{t_{Q_{\left.1_{(}\right)}}}^{i}, \lambda_{t_{Q_{2}\left(p_{j}\right)}}^{i}\right\}\right\} .
\end{aligned}
$$

With the consideration of the right side of equality, we have $T_{Q_{1}} \backslash T_{Q_{2}}=T_{V}$ and $T_{Q_{2}} \backslash T_{Q_{1}}=T_{W}$, where:

$$
\lambda_{t_{V}}^{i}\left(p_{j}\right)=\min \left\{\lambda_{t_{Q_{1\left(p_{j}\right)}}^{i}}^{i}, 1-\lambda_{t_{Q_{2\left(p_{j}\right)}}^{i}}^{i}\right\}
$$

and:

$$
\lambda_{t_{W}}^{i}\left(p_{j}\right)=\min \left\{\lambda_{t_{Q_{2}\left(p_{j}\right)}}^{i}, 1-\lambda_{t_{Q_{1\left(p_{j}\right)}}}^{i}\right\} .
$$

For $T_{Z}=T_{V} \sqcap T_{W}$, from Definition 3.6, we obtain that:

$$
\begin{aligned}
\lambda_{t_{Z}}^{i}\left(p_{j}\right)= & \max \left\{\lambda_{t_{V_{\left(p_{j}\right)}}}^{i}, \lambda_{t_{W_{\left(p_{j}\right)}}}^{i}\right\}= \\
& \max \left\{\min \left\{\lambda_{t_{Q_{1\left(p_{j}\right)}}}^{i}, 1-\lambda_{t_{Q_{2}\left(p_{j}\right)}}^{i}\right\},\right. \\
& \left.\min \left\{\lambda_{t_{Q_{2\left(p_{j}\right)}}}^{i}, 1-\lambda_{t_{Q_{1}\left(p_{j}\right)}}^{i}\right\}\right\} .
\end{aligned}
$$

We know that $\lambda_{t_{Q_{1}\left(p_{j}\right)}}^{i}, \lambda_{t_{Q_{2}\left(p_{j}\right)}}^{i} \in\left\{0, \frac{1}{2}, 1\right\}$ for all $p_{j} \in$ $\mathcal{P}$, and $\lambda_{t_{U}}^{i}\left(p_{j}\right)=\lambda_{t_{Z}}^{i}\left(p_{j}\right)$. This completes the proof. $\square$

Definition 3.9. Let $T_{Q_{1}}, T_{Q_{2}} \in \mathcal{T} \mathcal{V S S}(\mathfrak{X}, \mathcal{P})$. Then, the And-product of $T_{Q_{1}}$ and $T_{Q_{2}}$, denoted by $T_{Q_{1}} \bar{\wedge} T_{Q_{2}}$, is defined by the mapping $t_{Q_{1} \wedge Q_{2}}: \mathcal{P} \times \mathcal{P} \rightarrow \mathfrak{X}^{\left(\left\{0, \frac{1}{2}, 1\right\}\right)}$ such that:

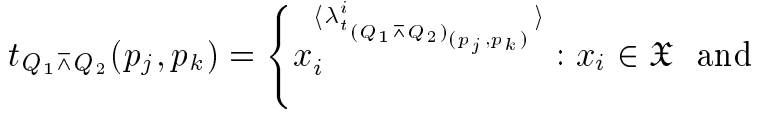

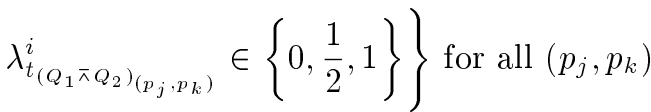

$$
\begin{aligned}
& \in \mathcal{P} \times \mathcal{P},
\end{aligned}
$$

where:

$$
\lambda_{t_{\left(Q_{1} \wedge Q_{2}\right)_{\left(p_{j}, p_{k}\right)}}^{i}}^{i}=\min \left\{\lambda_{t_{Q_{1\left(p_{j}\right)}}}^{i}, \lambda_{t_{Q_{\left.2_{(} p_{k}\right)}}^{i}}^{i}\right\} .
$$

Definition 3.10. Let $T_{Q_{1}}, T_{Q_{2}} \in \mathcal{T} \mathcal{V S S}(\mathfrak{X}, \mathcal{P})$. Then, the Or-product of $T_{Q_{1}}$ and $T_{Q_{2}}$, denoted by $T_{Q_{1}} \vee T_{Q_{2}}$, is defined by the mapping $t_{Q_{1} \underline{\vee} Q_{2}}: \mathcal{P} \times \mathcal{P} \rightarrow \mathfrak{X}^{\left(\left\{0, \frac{1}{2}, 1\right\}\right)}$ such that:

$$
\begin{aligned}
& t_{Q_{1} \vee Q_{2}}\left(p_{j}, p_{k}\right)=\left\{x_{i}^{\left\langle\lambda_{t\left(Q_{1} \vee Q_{2}\right)_{\left(p_{j}, p_{k}\right)}^{i}}^{i}\right\rangle}: x_{i} \in \mathfrak{X}\right. \text { and } \\
&\left.\lambda_{t_{\left(Q_{1} \vee Q_{2}\right)_{\left(p_{j}, p_{k}\right)}}^{i}}^{i} \in\left\{0, \frac{1}{2}, 1\right\}\right\}
\end{aligned}
$$

for all $\left(p_{j}, p_{k}\right) \in \mathcal{P} \times \mathcal{P}$, where:

$$
\lambda_{t_{\left(Q_{1} \vee Q_{2}\right)_{\left(p_{j}, p_{k}\right)}}^{i}}^{i}=\max \left\{\lambda_{t_{\left.Q_{\left.1_{(}\right)}\right)}}^{i}, \lambda_{t_{\left.Q_{2_{(}}\right)}}^{i}\right\} .
$$

Proposition 3.8. Let $T_{Q_{1}}, T_{Q_{2}}, T_{Q_{3}} \in \mathcal{T} \mathcal{V S S}(\mathfrak{X}, \mathcal{P})$. For all $*, \circ \in\{\bar{\wedge}, \underline{\vee}\}$ :

i. $T_{Q_{1}} *\left(T_{Q_{2}} * T_{Q_{3}}\right)=\left(T_{Q_{1}} * T_{Q_{2}}\right) * T_{Q_{3}}$;

ii. $T_{Q_{1}} *\left(T_{Q_{2}} \circ T_{Q_{3}}\right)=\left(T_{Q_{1}} * T_{Q_{2}}\right) \circ\left(T_{Q_{1}} * T_{Q_{3}}\right)$;

iii. $\left(T_{Q_{1}} \star T_{Q_{2}}\right) \circ T_{Q_{3}}=\left(T_{Q_{1}} \circ T_{Q_{3}}\right) \star\left(T_{Q_{2}} \circ T_{Q_{3}}\right)$.

Proof. They can be shown in a similar way to the proofs of Proposition 3.5.

Proposition 3.9. Let $T_{Q_{1}}, T_{Q_{2}} \in \mathcal{T} \mathcal{V S S}(\mathfrak{X}, \mathcal{P})$. Then, the following De Morgan's laws are held: 
i. $\overline{\left(T_{Q_{1}} \bar{\wedge} T_{Q_{2}}\right)}=\overline{T_{Q_{1}}} \vee \overline{T_{Q_{2}}}$;

ii. $\overline{\left(T_{Q_{1}} \vee T_{Q_{2}}\right)}=\overline{T_{Q_{1}}} \bar{\wedge} \overline{T_{Q_{2}}}$.

Proof. The proofs are similar to those of Proposition 3.6. $\square$

\section{Choice value of an object in three-valued soft $\operatorname{set}(\mathrm{s})$}

In 2002, Maji et al. [32] defined the choice value of an object in a soft set and concluded that this indication could be used to prioritize the objects in the soft set during the decision-making process. In addition, the idea of choice value initiated by Maji et al. was used for the three-valued soft sets.

In this part, it is taken $J=\{1,2, \ldots,|\mathcal{P}|\}$ where $|\mathcal{P}|$ denotes the cardinality of the parameter set $\mathcal{P}$.

Definition 4.1. Let $\mathfrak{X}$ be a set of alternatives (objects).

1. The choice value of an object $x_{i} \in \mathfrak{X}$ in the threevalued soft set $T_{Q}$ is defined and denoted by:

$$
\alpha_{i}=\sum_{j \in J}\left(x_{i j}\right)^{\xi}
$$

where $x_{i j}$ for all $i, j$ are the entries in the table of the three-valued soft set $T_{Q}$. Further, the arbitrary number $\xi \in \mathbb{R}^{+}$represents the overall impact coefficient of "undetermined" on the choice value.

2 . The choice value of an object $x_{i} \in \mathfrak{X}$ in the threevalued soft sets $T_{Q_{k}}$ for $k=1,2, \ldots, s$ is defined and denoted by:

$$
\alpha_{i}=\frac{\sum_{j \in J}\left(\left(x_{i j}^{\sqcap}\right)^{\xi}+\left(x_{i j}^{\sqcup}\right)^{\xi}\right)}{2},
$$

where $x_{i j}^{\sqcap}$ and $x_{i j}^{\sqcup}$ for all $i, j$ are the entries in the tables of three-valued soft sets $\bar{\Pi}_{k=1}^{s} T_{Q_{k}}$ and $\bigsqcup_{k=1}^{s} T_{Q_{k}}$, respectively. Also, the arbitrary number $\xi \in \mathbb{R}^{+}$is the overall impact coefficient of "undetermined" on the choice value.

Remark: It is clear that for $x_{i j}=\frac{1}{2},\left(x_{i j}\right)^{\xi} \rightarrow 1$ when $\xi \rightarrow 0$ and $\left(x_{i j}\right)^{\xi} \rightarrow 0$ when $\xi \rightarrow+\infty$. Take Figure 2 as an example. In case of minor modification, it is more appropriate to consider $0<\xi<1$. However, if the modification is major, and should consider $\xi \in$ $(1,+\infty)$.

\section{Algorithm 1: Selection}

Step 1. Choose feasible subsets $Q_{k}(k=1,2, \ldots, s)$ of the parameter set $\mathcal{P}$;

Step 2. Create the three-valued soft sets $T_{Q_{k}}$ for parameter subsets $Q_{k}(k=1,2, \ldots, s)$;

Step 3. Specify the overall impact coefficient of "undetermined" on the choice value, i.e., $\xi \in \mathbb{R}^{+}$;

\section{Step 4.}

- If $k>1$, obtain the intersection and union of threevalued soft sets $T_{Q_{k}}(k=1,2, \ldots, s)$;

- If $k=1$, skip to Step 4 .

Step 5. Calculate $\alpha_{i}$ for all $i$ 's;

Step 6. Find $l$, for which $\alpha_{l}=\max \alpha_{i}$.

Then, $x_{l}$ is the optimal choice object. If $l$ has more than one value, any one of them could be chosen.

Example 4.1. As an implementation of Algorithm 1, we attempt to solve our numerical problem in Example 3.3. According to Tables 8 and 9 , we have $\max \alpha_{i}=\alpha_{4}$ for $\xi=1, \frac{1}{4}, \frac{3}{2}, \sqrt{13}, 10$; then, the investment $x_{4}$ is an

Table 8. The choice value for three valued soft set $T_{Q_{1}}$ in Example 3.3.

\begin{tabular}{cccccccccc}
\hline & & & & & \multicolumn{5}{c}{$\boldsymbol{\alpha}_{\boldsymbol{i}}=\sum_{j}\left(\boldsymbol{x}_{\boldsymbol{i}}\right)^{\boldsymbol{\xi}}$} \\
\cline { 5 - 10 } $\mathfrak{X} / \mathcal{P}$ & $\boldsymbol{p}_{\mathbf{1}}$ & $\boldsymbol{p}_{\mathbf{2}}$ & $\boldsymbol{p}_{\mathbf{3}}$ & $\boldsymbol{p}_{\mathbf{4}}$ & $\boldsymbol{\xi}=\mathbf{1}$ & $\boldsymbol{\xi}=\frac{\mathbf{1}}{\mathbf{4}}$ & $\boldsymbol{\xi}=\frac{\mathbf{3}}{\mathbf{2}}$ & $\boldsymbol{\xi}=\sqrt{\mathbf{1 3}}$ & $\boldsymbol{\xi}=\mathbf{1 0}$ \\
\hline$x_{1}$ & 1 & 0 & 1 & 0 & 2 & 2 & 2 & 2 & 2 \\
$x_{2}$ & 0 & 1 & $\frac{1}{2}$ & 0 & 1.5 & 1.8408 & 1.3535 & 1.0824 & 1.0009 \\
$x_{3}$ & 1 & $\frac{1}{2}$ & $\frac{1}{2}$ & 0 & 2 & 2.6816 & 1.707 & 1.1648 & 1.0018 \\
$x_{4}$ & $\frac{1}{2}$ & 1 & 1 & 0 & 2.5 & 2.8408 & 2.3535 & 2.0824 & 2.0009 \\
\hline
\end{tabular}

\begin{tabular}{|c|c|c|c|c|}
\hline & $\begin{array}{c}\text { Ranking order of } \\
\text { choice value } \alpha_{i}\end{array}$ & $\begin{array}{c}\text { ranking preference } \\
\text { order of } x_{i}\end{array}$ & $\max \alpha_{i}$ & $\begin{array}{c}\text { Optimal choice } \\
\text { object }\end{array}$ \\
\hline$\xi=1$ & $\alpha_{4}>\alpha_{1}=\alpha_{3}>\alpha_{2}$ & $x_{4} \succ x_{1} \approx x_{3} \succ x_{2}$ & $\alpha_{4}$ & $x_{4}$ \\
\hline$\xi=\frac{1}{4}$ & $\alpha_{4}>\alpha_{3}>\alpha_{1}>\alpha_{2}$ & $x_{4} \succ x_{3} \succ x_{1} \succ x_{2}$ & $\alpha_{4}$ & $x_{4}$ \\
\hline$\xi=\frac{3}{2}$ & $\alpha_{4}>\alpha_{1}>\alpha_{3}>\alpha_{2}$ & $x_{4} \succ x_{1} \succ x_{3} \succ x_{2}$ & $\alpha_{4}$ & $x_{4}$ \\
\hline$\xi=\sqrt{13}$ & $\alpha_{4}>\alpha_{1}>\alpha_{3}>\alpha_{2}$ & $x_{4} \succ x_{1} \succ x_{3} \succ x_{2}$ & $\alpha_{4}$ & $x_{4}$ \\
\hline$\xi=10$ & $\alpha_{4}>\alpha_{1}>\alpha_{3}>\alpha_{2}$ & $x_{4} \succ x_{1} \succ x_{3} \succ x_{2}$ & $\alpha_{4}$ & $x_{4}$ \\
\hline
\end{tabular}

Table 9. The ranking preference order of objects for three valued soft set $T_{Q_{1}}$ in Example 3.3. 
optimal choice to invest some money.

Example 4.2. We consider three-valued soft sets $T_{Q_{1}}$ in Example 3.3 and $T_{Q_{2}}$ in Example 3.5. Then, we should make a common decision based on the data in these two three-valued soft sets for $\xi=2$. We obtain the intersection and union of $T_{Q_{1}}$ and $T_{Q_{2}}$ as in Tables 10 and 11, respectively.

According to Tables 10 and 11, we have $\max \alpha_{i}=$ $\alpha_{1}=\alpha_{4}=2.125$. It can be concluded that any of the investments $x_{1}$ and $x_{4}$ can be an optimal choice to invest some money.

In the decision-making process, all parameters of a parameter set may not be of equal importance. In such cases, weights can be imposed on the choice parameters; in other words, there is a weight $\omega_{j} \in(0,1]$ corresponding to each parameter $p_{j} \in Q$. If $p_{j} \in \mathcal{P}-Q$, we know that $\omega_{j}=0$. Generally, the total weight is $\sum_{j} \omega_{j}=1$. Now, let us describe the weighted choice value of an object in the structures of (weighted) threevalued soft sets.

Definition 4.2. Let $\mathfrak{X}$ be a set of alternatives (objects). Also, $T_{Q}$ is a (weighted) three-valued soft set over $\mathfrak{X}$ :

1. The weighted choice value of an object $x_{i} \in \mathfrak{X}$ in the (weighted) three-valued soft set $T_{Q}$ is defined and denoted by:

$$
\alpha_{i}^{\omega}=\sum_{j \in J} \omega_{j} \times\left(x_{i j}\right)^{\xi},
$$

where the arbitrary number $\xi \in \mathbb{R}^{+}$represents the overall impact coefficient of "undetermined" on the choice value. Also, $\omega_{j}$ denotes the weight corresponding to each parameter $p_{j}$ in the structure of three-valued soft set $T_{Q}$.

2. The weighted choice value of an object $x_{i} \in \mathfrak{X}$ in the (weighted) three-valued soft sets $T_{Q_{k}}$ for $k=$ $1,2, \ldots, s$ is defined and denoted by:

Table 10. Three valued soft set $T_{Q_{1}} \bar{\Pi} T_{Q_{2}}$.

\begin{tabular}{cccccc}
\hline $\mathfrak{X} / \mathcal{P}$ & $\boldsymbol{p}_{\mathbf{1}}$ & $\boldsymbol{p}_{\mathbf{2}}$ & $\boldsymbol{p}_{\mathbf{3}}$ & $\boldsymbol{p}_{\boldsymbol{4}}$ & $\sum_{\boldsymbol{j}}\left(\boldsymbol{x}_{\boldsymbol{i} \boldsymbol{j}}^{\Gamma}\right)^{\mathbf{2}}$ \\
\hline$x_{1}$ & $\frac{1}{2}$ & 0 & 1 & 0 & 1.25 \\
$x_{2}$ & 0 & 0 & 0 & 0 & 0 \\
$x_{3}$ & $\frac{1}{2}$ & $\frac{1}{2}$ & $\frac{1}{2}$ & 0 & 0.75 \\
$x_{4}$ & $\frac{1}{2}$ & 0 & 0 & 0 & 0.25 \\
\hline
\end{tabular}

Table 11. Three valued soft set $T_{Q_{1}} \underline{T_{Q_{2}}}$.

\begin{tabular}{cccccc}
\hline $\mathfrak{X} / \mathcal{P}$ & $\boldsymbol{p}_{\mathbf{1}}$ & $\boldsymbol{p}_{\boldsymbol{2}}$ & $\boldsymbol{p}_{\boldsymbol{3}}$ & $\boldsymbol{p}_{\boldsymbol{4}}$ & $\sum_{\boldsymbol{j}}\left(\boldsymbol{x}_{\boldsymbol{i} \boldsymbol{j}}^{\sqcup}\right)^{\mathbf{2}}$ \\
\hline$x_{1}$ & 1 & 1 & 1 & 0 & 3 \\
$x_{2}$ & 0 & 1 & $\frac{1}{2}$ & 0 & 1.25 \\
$x_{3}$ & 1 & $\frac{1}{2}$ & 1 & 0 & 2.25 \\
$x_{4}$ & 1 & 1 & 1 & 1 & 4 \\
\hline
\end{tabular}

$$
\alpha_{i}^{\omega}=\frac{\sum_{j \in J} \omega_{j}^{\text {ort }} \times\left(\left(x_{i j}^{\sqcap}\right)^{\xi}+\left(x_{i j}^{\sqcup}\right)^{\xi}\right)}{2},
$$

where the arbitrary number $\xi \in \mathbb{R}^{+}$is the overall impact coefficient of "undetermined" on the choice value. Also:

$$
\omega_{j}^{\text {ort }}=\frac{\sum_{k=1}^{s} \omega_{j}^{k}}{s},
$$

where $\omega_{j}^{k}$ indicates the weight corresponding to each parameter $p_{j}$ in the structure of three-valued soft set $T_{Q_{k}}$.

\section{Algorithm 2: Selection by imposing weights on parameters}

Step 1. Choose the feasible subsets $Q_{k}(k=$ $1,2, \ldots, s)$ of the parameter set $\mathcal{P}$ and determine its weights (i.e., $\left.\omega^{k}\right)$ for each subsets $Q_{k}$;

Step 2. Create the (weighted) three-valued soft sets $T_{Q_{k}}$ for the parameter subsets $Q_{k}(k=1,2, \ldots, s)$;

Step 3. Specify the overall impact coefficient of "undetermined" on the choice value, i.e., $\xi \in \mathbb{R}^{+}$;

\section{Step 4.}

- If $k>1$, obtain the intersection and union of threevalued soft sets $T_{Q_{k}}(k=1,2, \ldots, s)$, and

- If $k=1$, skip to Step 4 .

Step 5. Calculate $\alpha_{i}^{\omega}$ for all $i$ 's;

Step 6. Find $l$ for which $\alpha_{l}^{\omega}=\max \alpha_{i}^{\omega}$.

Then, $x_{l}$ is the optimal choice object. If $l$ has more than one value, any one of them could be chosen.

Example 4.3. the numerical problem proposed in Example 3.3 was taken into consideration. Also, the following weights were measured for the parameters of $Q_{1}: \omega_{1}^{1}=0.6$ for the parameter $p_{1}=$ high returns, $\omega_{2}^{1}=0.3$ for the parameter $p_{2}=$ low risk and $\omega_{3}^{1}=0.1$ for the parameter $p_{3}=$ high security. Since $p_{4} \notin Q_{1}$, the weight of parameter $p_{4} \in \mathcal{P}$ can be considered " 0 ".

According to Table 12, we have $\max \alpha_{i}^{\omega}=\alpha_{3}^{\omega}$ (for $\xi=1, \frac{1}{10}, 3$ ). Then, the optimal choice is $x_{3}$.

Example 4.4. Consider the weighted three-valued soft sets $T_{Q_{1}}$ in Example 4.3. Also, we take the following parameter weights for $T_{Q_{2}}$ in Table $4: \omega_{1}^{2}=\omega_{4}^{2}=0.2$ and $\omega_{2}^{2}=\omega_{3}^{2}=0.3$. For $\xi=1$, we obtain weighted choice values of alternatives $x_{i}(i=1,2,3,4)$ as $\alpha_{1}^{\omega}=$ $0.65, \alpha_{2}^{\omega}=0.2, \alpha_{3}^{\omega}=0.6, \alpha_{4}^{\omega}=0.6$. Since $\max \alpha_{i}^{\omega}=$ $\alpha_{1}^{\omega}=0.65$, the optimal choice is $x_{1}$.

In Algorithms 1 and 2, for each decision-maker, the overall impact coefficient $(\xi)$ of "undetermined" on the choice value is taken the same. These two algorithms cannot be used if each decision-maker selects the impact coefficient of "undetermined" differently. To address these shortcomings, we will create new decision-making algorithms. 
Table 12. The weighted choice value for three valued soft set $T_{Q_{1}}$ in Example 3.3

\begin{tabular}{ccccccccc}
\hline & & & & & \multicolumn{2}{c}{$\boldsymbol{\alpha}_{\boldsymbol{i}}^{\boldsymbol{\omega}}=\sum_{j} \boldsymbol{\omega}_{j}^{\mathbf{1}} \times\left(\boldsymbol{x}_{\boldsymbol{i j}}\right)^{\boldsymbol{\xi}}$} \\
\cline { 6 - 8 } $\mathfrak{X} / \mathcal{P}$ & $\boldsymbol{p}_{\mathbf{1}}\left(\boldsymbol{\omega}_{\mathbf{1}}^{\mathbf{1}}=\mathbf{0 . 6}\right)$ & $\boldsymbol{p}_{\mathbf{2}}\left(\boldsymbol{\omega}_{\mathbf{2}}^{\mathbf{1}}=\mathbf{0 . 3}\right)$ & $\boldsymbol{p}_{\mathbf{3}}\left(\boldsymbol{\omega}_{\mathbf{3}}^{\mathbf{1}}=\mathbf{0 . 1}\right)$ & $\boldsymbol{p}_{\mathbf{4}}\left(\boldsymbol{\omega}_{4}^{\mathbf{1}}=\mathbf{0}\right)$ & $\boldsymbol{\xi}=\mathbf{1}$ & $\boldsymbol{\xi}=\frac{\mathbf{1}}{\mathbf{1 0}}$ & $\boldsymbol{\xi}=\mathbf{3}$ \\
\hline$x_{1}$ & 1 & 0 & 1 & 0 & 0.7 & 0.7 \\
$x_{2}$ & 0 & 1 & $\frac{1}{2}$ & 0 & 0.75 & 0.3933 & 0.3125 \\
$x_{3}$ & 1 & $\frac{1}{2}$ & 1 & 0 & 0.8 & 0.9732 & 0.65 \\
$x_{4}$ & $\frac{1}{2}$ & 1 & 1 & 0 & 0.7 & 0.9598 & 0.475 \\
\hline
\end{tabular}

\section{Three-valued soft decision-making model based on TOPSIS}

This section primarily focuses on the TOPSIS, which produces satisfactory results during decision-making. Here, this technique was rebuilt to deal with the multi-criteria-group decision-making problems based on three-valued soft sets, thus constructing a novel decision-making model.

TOPSIS is an approach to solving multi-criteria decision-making problems based on a decision maker. In 2007, Shih et al. [47] extended this method for group decision-making. The operations in the process of TOPSIS include decision matrix normalization, distance measures, and aggregation operators [47]. Generally, a decision matrix is required prior to the beginning of the process. As a result of this process, the output data are interpreted so that the ranking order of alternatives can be obtained. In summary, the TOPSIS approach is a practical and useful method for ranking and selecting a number of externally determined alternatives through distance measures. The main procedure of TOPSIS is given in a series of steps (see [48-51]).

Now, a multi-criteria group decision-making model is proposed using the TOPSIS on three-valued soft sets.

\section{Algorithm 3: TOPSIS based three-valued soft sets}

Step 1. The multi-criteria group decision-making problem is identified. In this step, decision makers (experts), alternatives, and choice parameters are determined. Suppose that $D M=\left\{E_{k}: k \in I_{s}=\right.$ $\{1,2, \ldots, s\}\}$ is a set of decision makers (experts) and $E_{k}$ denotes the $k$ th decision maker (expert). Also, $x_{i}$ $\left(i \in \mathcal{I}_{m}=\{1,2, \ldots, m\}\right)$ denotes the $i$ th alternative, and $p_{j}\left(j \in \mathcal{I}_{n}=\{1,2, \ldots, n\}\right)$ represents the $j$ th parameter (criterion or attribute).

Considering these data, each decision maker $E_{k}\left(k \in I_{s}\right)$ create three-valued soft set $T_{Q_{k}}$ and measures the weights of parameters as $\omega_{j}^{k}\left(j \in \mathcal{I}_{n}\right)$ satisfying the condition $\sum_{j=1}^{n} \omega_{j}^{k}=1$.

Moreover, each decision maker $E_{k}$ specifies the impact coefficient of "undetermined" in decisionmaking, i.e., $\xi_{k}$;
Step 2. For each decision maker $E_{k}$, the decision matrix $\mathfrak{D}^{k}$ is constructed and represented as follows:

$$
\begin{aligned}
& \begin{array}{llllll}
p_{1} & p_{2} & \cdot & \cdot & p_{n}
\end{array} \\
& \mathfrak{D}^{k}=\begin{array}{c}
x_{1} \\
x_{2} \\
\cdot \\
\cdot \\
\cdot \\
x_{m}
\end{array}\left[\begin{array}{cccccc}
d_{11}^{k} & d_{12}^{k} & . & . & . & d_{1 n}^{k} \\
d_{21}^{k} & d_{22}^{k} & . & . & . & d_{2 n}^{k} \\
\cdot & \cdot & \cdot & & & \cdot \\
\cdot & \cdot & & \cdot & & \cdot \\
\dot{d} & \cdot & & & \cdot & \cdot \\
d_{m 1}^{k} & d_{m 2}^{k} & . & . & . & d_{m n}^{k}
\end{array}\right]=\left[d_{i j}^{k}\right]_{m \times n},
\end{aligned}
$$

where $d_{i j}^{k}=\left(x_{i j}^{k}\right)^{\xi_{k}}$ that $x_{i j}^{k}$ for all $i, j$ are the entries in the table of three-valued soft set $T_{Q_{k}}$;

Step 3. After constructing the decision matrices, these are normalized (standardized).

For each decision matrix $\mathfrak{D}^{k}$, the normalized decision matrix $\mathfrak{R}^{k}$ is constructed and expressed as follows:

$$
\mathfrak{R}^{k}=\left[\begin{array}{cccccc}
r_{11}^{k} & r_{12}^{k} & \cdot & \cdot & \cdot & r_{1 n}^{k} \\
r_{21}^{k} & r_{22}^{k} & \cdot & \cdot & \cdot & r_{2 n}^{k} \\
\cdot & \cdot & \cdot & & & \cdot \\
\cdot & \cdot & & \cdot & & \cdot \\
\cdot & \cdot & & \cdot & \cdot \\
r_{m 1}^{\dot{k}} & r_{m 2}^{k} & \cdot & \cdot & \cdot & r_{m n}^{\dot{k}}
\end{array}\right]=\left[r_{i j}^{k}\right]_{m \times n}
$$

where:

$$
r_{i j}^{k}= \begin{cases}\frac{d_{i j}^{k}}{\sqrt{\sum_{\ell=1}^{m}\left(d_{\ell j}^{k}\right)^{2}}}, & \text { if } d_{i j}^{k} \neq 0 \\ 0, & \text { if } d_{i j}^{k}=0\end{cases}
$$

for all $k \in \mathcal{I}_{s}, i \in \mathcal{I}_{m}$, and $j \in \mathcal{I}_{n}$.

Step 4. Given different weights of parameters for each decision-maker, the weighted normalized decision matrix is calculated by multiplying the weights of evaluation parameters by values in the normalized decision matrix.

For each normalized decision matrix $\mathfrak{R}^{k}$, the weighted normalized decision matrix $\mathrm{V}^{k}$ is created as follows:

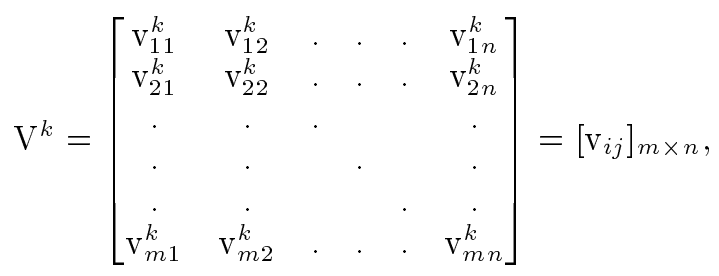


where:

$$
\mathrm{v}_{i j}^{k}=w_{j}^{k} \times r_{i j}^{k}
$$

for all $k \in \mathcal{I}_{s}, i \in \mathcal{I}_{m}$ and $j \in \mathcal{I}_{n}$.

Step 5. By combining the weighted normalized decision matrices $\mathrm{V}^{k}\left(k \in \mathcal{I}_{s}\right)$, the average weighted normalized decision matrix $\mathcal{V}$ can be obtained. follows:

The structure of the matrix $\mathcal{V}$ is expressed as

$$
\mathcal{V}=\left[\begin{array}{cccccc}
v_{11} & v_{12} & \cdot & \cdot & \cdot & v_{1 n} \\
v_{21} & v_{22} & \cdot & \cdot & \cdot & v_{2 n} \\
\cdot & \cdot & \cdot & & & \cdot \\
\cdot & \cdot & & \cdot & & \cdot \\
\cdot & \cdot & & & \cdot & \cdot \\
v_{m 1} & v_{m 2} & \cdot & \cdot & \cdot & v_{m n}
\end{array}\right]=\left[v_{i j}\right]_{m \times n}
$$

where for all $i \in \mathcal{I}_{m}$ and $j \in \mathcal{I}_{n}$ :

$$
v_{i j}=\mathrm{v}_{i j}^{1} \oplus \mathrm{v}_{i j}^{2} \oplus \ldots \oplus \mathrm{v}_{i j}^{s} \text {. }
$$

In other words, the value $v_{i j}$ is obtained by combining the weighted normalized decision value $\mathrm{v}_{i j}^{k}$ through an operation $\oplus$. Here, the operation $\oplus$ can offer many choices: arithmetic mean, geometric mean, harmonic mean and their modification.

Note: In this study, we will take the arithmetic mean of all individual measures.

Step 6. The positive and negative ideal solutions $\mathcal{V}^{\top}$ and $\mathcal{V}^{\perp}$ are determined using the average weighted normalized decision matrix $\mathcal{V}$.

In the TOPSIS approach, the parameters (criteria or attributes) are evaluated in terms of benefit (cf. Example 3.3) and cost (cf. Example 3.1). Suppose that $\mathcal{J}_{1}$ and $\mathcal{J}_{2}$ are the sets of benefit and cost parameters, respectively, where $\mathcal{J}_{1} \cap \mathcal{J}_{2}=\phi$ and $\mathcal{J}_{1} \cup \mathcal{J}_{2}=\{1,2, \ldots, n\} . \mathcal{V}^{\top}$ and $V_{L}$ are described as follows:

- $\mathcal{V}^{\top}$ is the set which shows that the most suitable alternative for each parameter may be preferred (PIS). This set is obtaibed and shown in the following:

$$
\begin{aligned}
\mathcal{V}^{\top} & =\left\{v_{1}^{\top}, v_{2}^{\top}, \ldots, v_{j}^{\top}, \ldots, v_{n}^{\top}\right\} \\
& =\left\{\left(\max _{i} v_{i j}^{\perp}: j \in \mathcal{J}_{1}\right),\left(\min _{i} v_{i j}^{\perp}: j \in \mathcal{J}_{2}\right),\right. \\
& \left.i \in \mathcal{I}_{m}\right\} .
\end{aligned}
$$

- $\mathcal{V}^{\perp}$ is the set showing the least preferable alternative for each parameter (NIS). This set is obtained as follows:

$$
\begin{aligned}
\mathcal{V}^{\perp} & =\left\{v_{1}^{\perp}, v_{2}^{\perp}, \ldots, v_{j}^{\perp}, \ldots, v_{n}^{\perp}\right\} \\
& =\left\{\left(\min _{i} v_{i j}^{\perp}: j \in \mathcal{J}_{1}\right),\left(\max _{i} v_{i j}^{\perp}: j \in \mathcal{J}_{2}\right),\right. \\
& \left.i \in \mathcal{I}_{m}\right\} .
\end{aligned}
$$

Step 7. The separation measurements of alternatives to the ideal solutions are obtained through the Euclidean distance formula.

The separation measurement of each alternative $x_{i}$ to the positive ideal solution $\mathcal{V}^{\top}$ is calculated as follows:

$$
\mathcal{S}_{i}^{\top}=\sqrt{\sum_{j=1}^{n}\left(v_{i j}-v_{j}^{\top}\right)^{2}} .
$$

The separation measurement of each alternative $x_{i}$ to the negative ideal solution $\mathcal{V}^{\perp}$ is calculated as follows:

$$
\mathcal{S}_{i}^{\perp}=\sqrt{\sum_{j=1}^{n}\left(v_{i j}-v_{j}^{\perp}\right)^{2}} .
$$

Here, $\mathcal{S}_{i}^{\top}$ and $\mathcal{S}_{i}^{\perp}$ represent the distance of the alternative $x_{i}$ from PIS and NIS, respectively.

Step 8. The relative closeness of each alternative to the ideal solutions is also calculated.

The relative closeness $\mathcal{C}_{i}^{\dagger}$ of the alternatives $x_{i}$ with respect to the ideal solutions can be expressed as:

$$
\mathcal{C}_{i}^{\dagger}=\frac{\mathcal{S}_{i}^{\perp}}{\mathcal{S}_{i}^{\perp}+\mathcal{S}_{i}^{\top}}, \forall i \in \mathcal{I}_{m} \quad\left(0 \leq \mathcal{C}_{i}^{\dagger} \leq 1\right) .
$$

Step 9. The alternatives (objects) are ranked in order of preference.

A set of alternatives $x_{i}$ can be ranked according to the descending order of the values $\mathcal{C}_{i}^{\dagger}$.

To show the potential of the proposed approach, a reallife practice was suggested, adopted from Figure 4.

Example 5.1. Assume that two experts are about to determine the best mobile brand by examining the new model mobile phones presented by six different mobile phone brands. The first expert $E_{1}$ reviews each brand's mobile phone with memory of $128 \mathrm{~GB}$, and the second expert $E_{2}$ reviews each brand's mobile phone with memory of 64 GB. Let $\mathfrak{X}=\left\{x_{1}, x_{2}, x_{3}, x_{4}, x_{5}, x_{6}\right\}$ be a set of six different mobile phone brands. Also, the set of parameters is employed to determine the brand with the best mobile phones, which is given as $\mathcal{P}=\left\{p_{1}, p_{2}, p_{3}, p_{4}, p_{5}, p_{6}, p_{7}\right\}$ where $p_{1}=$ optical image stabilization, $p_{2}=$ quick charging, $p_{3}=$ expandable memory, $p_{4}=$ waterproof , $p_{5}=$ autofocus, $p_{6}=$ cheap, and $p_{7}=$ fingerprint sensor. Each of these experts proceeds to the decision making stage after reviewing comparisons on mobile phones on a comparison-focused website (such as "www.91mobiles.com" and "www.smartprix.com").

To deal with this problem, the steps of Algorithm 3 are followed:

Step 1. The experts $E_{1}$ and $E_{2}$ determine the 
Table 13. The weights of choice parameters of experts $E_{1}$ and $E_{2}$.

\begin{tabular}{lcccccccc}
\hline & $\boldsymbol{\omega}_{\mathbf{1}}$ & $\boldsymbol{\omega}_{\mathbf{2}}$ & $\boldsymbol{\omega}_{\mathbf{3}}$ & $\boldsymbol{\omega}_{\mathbf{4}}$ & $\boldsymbol{\omega}_{\mathbf{5}}$ & $\boldsymbol{\omega}_{\mathbf{6}}$ & $\boldsymbol{\omega}_{\mathbf{7}}$ & \\
\hline First expert $\left(E_{1}\right)$ & 0.22 & 0.15 & 0.13 & 0.15 & 0.13 & 0.11 & 0.11 & $\sum_{j=1}^{7} \omega_{j}^{1}=1$ \\
Second expert $\left(E_{2}\right)$ & 0.24 & 0.12 & 0.12 & 0.16 & 0.14 & 0.1 & 0.12 & $\sum_{j=1}^{7} \omega_{j}^{2}=1$ \\
\hline
\end{tabular}

Table 14. Three valued soft sets of experts $E_{1}$ and $E_{2}$.

\begin{tabular}{|c|c|c|c|c|c|c|c|c|c|c|c|c|c|c|}
\hline \multirow{2}{*}{$\begin{array}{c}\text { Decision makers: } \\
\mathfrak{X} / \mathcal{P} \\
\end{array}$} & \multicolumn{7}{|c|}{ First expert $\left(E_{1}\right)$} & \multicolumn{7}{|c|}{ Second expert $\left(E_{2}\right)$} \\
\hline & $p_{1}$ & $p_{2}$ & $p_{3}$ & $p_{4}$ & $p_{5}$ & $p_{6}$ & $p_{7}$ & $p_{1}$ & $p_{2}$ & $p_{3}$ & $p_{4}$ & $p_{5}$ & $p_{6}$ & $p_{7}$ \\
\hline$x_{1}$ & 1 & 0 & 0 & $\frac{1}{2}$ & $\frac{1}{2}$ & 1 & 0 & $\frac{1}{2}$ & $\frac{1}{2}$ & 1 & 0 & $\frac{1}{2}$ & 1 & 0 \\
\hline$x_{2}$ & 1 & $\frac{1}{2}$ & $\frac{1}{2}$ & 1 & 1 & 1 & $\frac{1}{2}$ & 1 & 0 & $\frac{1}{2}$ & 1 & 1 & 1 & 1 \\
\hline$x_{3}$ & $\frac{1}{2}$ & 0 & $\frac{1}{2}$ & 1 & $\frac{1}{2}$ & 0 & 1 & $\frac{1}{2}$ & 0 & 0 & 1 & 1 & 0 & 1 \\
\hline$x_{4}$ & $\frac{1}{2}$ & $\frac{1}{2}$ & 1 & $\frac{1}{2}$ & $\frac{1}{2}$ & 1 & 1 & 0 & $\frac{1}{2}$ & 1 & 0 & 0 & 1 & $\frac{1}{2}$ \\
\hline$x_{5}$ & 0 & $\frac{1}{2}$ & $\frac{1}{2}$ & 1 & $\frac{1}{2}$ & 0 & 1 & $\frac{1}{2}$ & 0 & 0 & 1 & 0 & 1 & 1 \\
\hline$x_{6}$ & $\frac{1}{2}$ & $\frac{1}{2}$ & 1 & 1 & 0 & 1 & 1 & 1 & 1 & 1 & 1 & 0 & 1 & 1 \\
\hline Impact coefficient $\xi_{k}$ : & \multicolumn{7}{|c|}{$\xi_{1}=1.5$} & \multicolumn{7}{|c|}{$\xi_{2}=2$} \\
\hline
\end{tabular}

parameter sets as $Q_{1}=Q_{2}=\mathcal{P}$, respectively. In addition, they determine the weights of their choice parameters as $\omega_{j}^{k}$ for all $j=1,2, \ldots, 7$ and $k=1,2$. (see Table 13). The experts collect data about each brand's mobile phone $x_{i}(i=1,2, \ldots, 6)$ for each attribute $p_{j}$ and create three-valued soft sets in Table 14.

Step 2. According to Table 14, the decision matrices $\mathfrak{D}^{k}(k=1,2)$ are constructed in the equations shown in Box VI.

Step 3. For each decision matrix $\mathfrak{D}^{k}(k=1,2)$, the normalized decision matrix $\mathfrak{R}^{k}(k=1,2)$ is constructed in the equations shown in Box VII.

Step 4. For each normalized decision matrix $\mathfrak{R}^{k}$ $(k=1,2)$, the weighted normalized decision matrix $\mathrm{V}^{k}(k=1,2)$ is formed by the equations shown in Box VIII.

Step 5. Then, the average weighted normalized decision matrix is constructed by the equation shown in Box IX, where the operation $\oplus$ represents the arithmetic mean.

Step 6. The positive and negative ideal solutions $\mathcal{V}^{\top}$ and $\mathcal{V}^{\perp}$ are determined as follows:

$$
\begin{gathered}
\mathcal{V}^{\top}=\left\{v_{1}^{\top}=0.1524, v_{2}^{\top}=0.094, v_{3}^{\top}=0.0764,\right. \\
v_{4}^{\top}=0.0763, v_{5}^{\top}=0.1017, v_{6}^{\top}=0.0498, \\
\left.v_{7}^{\top}=0.0568\right\}
\end{gathered}
$$

$\mathfrak{D}^{1}=\left[\begin{array}{cccccccc}1 & 0 & 0 & 0.3535 & 0.3535 & 1 & 0 \\ 1 & 0.3535 & 0.3535 & 1 & 1 & 1 & 0.3535 \\ 0.3535 & 0 & 0.3535 & 1 & 0.3535 & 0 & 1 \\ 0.3535 & 0.3535 & 1 & 0.3535 & 0.3535 & 1 & 1 \\ 0 & 0.3535 & 0.3535 & 1 & 0.3535 & 0 & 1 \\ 0.3535 & 0.3535 & 1 & 1 & 0 & 1 & 1\end{array}\right]=\left[d_{i j}^{1}\right]_{6 \times 7}$,
$\mathfrak{D}^{2}=\left[\begin{array}{ccccccc}0.25 & 0.25 & 1 & 0 & 0.25 & 1 & 0 \\ 1 & 0 & 0.25 & 1 & 1 & 1 & 1 \\ 0.25 & 0 & 0 & 1 & 1 & 0 & 1 \\ 0 & 0.25 & 1 & 0 & 0 & 1 & 0.25 \\ 0.25 & 0 & 0 & 1 & 0 & 1 & 1 \\ 1 & 1 & 1 & 1 & 0 & 1 & 1\end{array}\right]=\left[d_{i j}^{2}\right]_{6 \times 7}$ 


$\begin{aligned} \mathfrak{R}^{1} & =\left[\begin{array}{ccccccc}0.6489 & 0 & 0 & 0.1714 & 0.2886 & 0.5 & 0 \\ 0.6489 & 0.5001 & 0.2293 & 0.485 & 0.8166 & 0.5 & 0.174 \\ 0.2293 & 0 & 0.2293 & 0.485 & 0.2886 & 0 & 0.4923 \\ 0.2293 & 0.5001 & 0.6489 & 0.1714 & 0.2886 & 0.5 & 0.4923 \\ 0 & 0.5001 & 0.2293 & 0.485 & 0.2886 & 0 & 0.4923 \\ 0.2293 & 0.5001 & 0.2293 & 0.485 & 0 & 0.5 & 0.4923\end{array}\right] \\ \mathfrak{R}^{2} & =\left[\begin{array}{ccccccc}0.169 & 0.2357 & 0.5714 & 0 & 0.0174 & 0.4472 & 0 \\ 0.6761 & 0 & 0.1428 & 0.5 & 0.6963 & 0.4472 & 0.4961 \\ 0.169 & 0 & 0 & 0.5 & 0.6963 & 0 & 0.4961 \\ 0 & 0.2357 & 0.5714 & 0 & 0 & 0.4472 & 0.124 \\ 0.169 & 0 & 0 & 0.5 & 0 & 0.4472 & 0.4961 \\ 0.6761 & 0.9428 & 0.5714 & 0.5 & 0 & 0.4472 & 0.4961\end{array}\right]\end{aligned}$

Box VII

$$
\begin{aligned}
\mathrm{V}^{1}= & {\left[\begin{array}{ccccccc}
0.1427 & 0 & 0 & 0.0257 & 0.0375 & 0.055 & 0 \\
0.1427 & 0.075 & 0.0298 & 0.0727 & 0.1061 & 0.055 & 0.0191 \\
0.0504 & 0 & 0.0298 & 0.0727 & 0.0375 & 0 & 0.0541 \\
0.0504 & 0.075 & 0.0843 & 0.0257 & 0.0375 & 0.055 & 0.0541 \\
0 & 0.075 & 0.0298 & 0.0727 & 0.0375 & 0 & 0.0541 \\
0.0504 & 0.075 & 0.0298 & 0.0727 & 0 & 0.055 & 0.0541
\end{array}\right], } \\
\mathrm{V}^{2}= & {\left[\begin{array}{ccccccc}
0.0405 & 0.0282 & 0.0685 & 0 & 0.0243 & 0.0447 & 0 \\
0.1622 & 0 & 0.0171 & 0.08 & 0.0974 & 0.0447 & 0.0595 \\
0.0405 & 0 & 0 & 0.08 & 0.0974 & 0 & 0.0595 \\
0 & 0.0282 & 0.0685 & 0 & 0 & 0.0447 & 0.0148 \\
0.0405 & 0 & 0 & 0.08 & 0 & 0.0447 & 0.0595 \\
0.1622 & 0.1131 & 0.0685 & 0.08 & 0 & 0.0447 & 0.0595
\end{array}\right] . }
\end{aligned}
$$

\section{Box VIII}

and:

$$
\begin{aligned}
\mathcal{V}^{\perp}= & \left\{v_{1}^{\perp}=0.0202, v_{2}^{\perp}=0, v_{3}^{\perp}=0.0149,\right. \\
& \left.v_{4}^{\perp}=0.0128, v_{5}^{\perp}=0, v_{6}^{\perp}=0, v_{7}^{\perp}=0\right\} .
\end{aligned}
$$

Step 7. The separation measurements $\mathcal{S}_{i}^{\top}$ and $\mathcal{S}_{i}^{\perp}$ of each alternative $x_{i}$ to the ideal solutions are given in Table 15.
Step 8. The relative closeness $\mathcal{C}_{i}^{\dagger}$ of each alternative to the ideal solutions is calculated as follows:

$$
\begin{array}{rlrl}
\mathcal{C}_{1}^{\dagger} & =0.3767, & \mathcal{C}_{2}^{\dagger}=0.7095, \\
\mathcal{C}_{3}^{\dagger}=0.399, & \mathcal{C}_{4}^{\dagger}=0.3706, \\
\mathcal{C}_{5}^{\dagger}=0.3513, & \mathcal{C}_{6}^{\dagger}=0.589 .
\end{array}
$$

Step 9. According to the descending order of

$$
\mathcal{V}=\mathrm{V}^{1} \oplus \mathrm{V}^{2}=\left[\begin{array}{ccccccc}
0.0916 & 0.0141 & 0.0342 & 0.0128 & 0.0309 & 0.0498 & 0 \\
0.1524 & 0.0375 & 0.0234 & 0.0763 & 0.1017 & 0.0498 & 0.0393 \\
0.0454 & 0 & 0.0149 & 0.0763 & 0.0674 & 0 & 0.0568 \\
0.0252 & 0.0516 & 0.0764 & 0.0128 & 0.0187 & 0.0498 & 0.0344 \\
0.0202 & 0.0375 & 0.0149 & 0.0763 & 0.0187 & 0.0223 & 0.0568 \\
0.1063 & 0.094 & 0.0491 & 0.0763 & 0 & 0.0498 & 0.0568
\end{array}\right]=\left[v_{i j}\right]_{6 \times 7}
$$


Table 15. Separation measurements $\mathcal{S}_{i}^{\top}$ and $\mathcal{S}_{i}^{\perp}$.

\begin{tabular}{ccccccc}
\hline & $\boldsymbol{x}_{\boldsymbol{1}}$ & $\boldsymbol{x}_{\boldsymbol{2}}$ & $\boldsymbol{x}_{\boldsymbol{3}}$ & $\boldsymbol{x}_{\boldsymbol{4}}$ & $\boldsymbol{x}_{\boldsymbol{5}}$ & $\boldsymbol{x}_{\boldsymbol{6}}$ \\
\hline $\mathcal{S}_{i}^{\top}$ & 0.1542 & 0.0787 & 0.167 & 0.1705 & 0.178 & 0.1144 \\
\hline $\mathcal{S}_{\boldsymbol{i}}^{\perp}$ & 0.0932 & 0.1923 & 0.1109 & 0.1004 & 0.0964 & 0.164 \\
\hline
\end{tabular}

the values $\mathcal{C}_{i}^{\dagger}$, the ranking order of alternatives is obtained below:

$$
x_{2} \succ x_{6} \succ x_{3} \succ x_{1} \succ x_{4} \succ x_{5}
$$

Then, it can be argued that $x_{2}$ is the best mobile phone brand according to the data presented by experts.

\section{Three-valued soft decision-making model based on ELECTRE}

This section introduces a modified version of ELECTRE technique ("ELimination Et Choix Traduisant la REalité" or "Elimination and Choice Expressing Reality"), which is generally intended to output choosing, sorting, and ranking, to deal with the multi-criteria group decision-making problems based on three-valued soft sets.

As was first applied in 1965, the ELECTRE method was employed to choose the best alternative(s) from a given set of alternatives and it was applied to three fundamental problems:

Choosing: Selecting a restricted number of the most interesting potential alternatives, as small as possible which will justify elimination of the others.

Sorting: Assigning each potential alternative to one of the categories a family previously described; the categories are ordered from the worst to best .

Ranking: Ordering alternatives from the best to worst with the possibility of ties.

The main procedure of ELECTRE is described in a series of steps (see [52-56].

Now, a multi-criteria group decision making model is constructed on three-valued soft sets using the modified ELECTRE technique.

\section{Algorithm 4: ELECTRE based three-valued soft sets}

Step 1. Describe the multi-criteria group-decisionmaking problem (the same as Step 1 in Algorithm 3).

Step 2. For each decision maker $E_{k}$, the decision matrix $\mathfrak{D}^{k}$ is constructed (the same as Step 2 in Algorithm 3).
Step 3. For each decision matrix $\mathfrak{D}^{k}$, the normalized decision matrix $\mathfrak{R}^{k}$ is constructed (the same as Step 3 in Algorithm 3).

Step 4. For each normalized decision matrix $\mathfrak{R}^{k}$, the weighted normalized decision matrix $\mathrm{V}^{k}$ is created (the same as Step 4 in Algorithm 3).

Step 5. After combining the weighted normalized decision matrices $\mathrm{V}^{k}\left(k \in \mathcal{I}_{s}\right)$, the average weighted normalized decision matrix $\mathcal{V}$ is formed (the same as Step 5 in Algorithm 3).

Step 6. The concordance sets and discordance sets are determined. The concordance set is composed of the index of all parameters for which the alternative $x_{\tau}$ is preferred over the alternative $x_{\kappa}$. This set can be described as follows.

For $\tau, \kappa \in \mathcal{I}_{m}$ and $\tau \neq \kappa$ (note that an alternative is not compared to itself):

$$
\mathcal{J}_{\tau \kappa}^{+}=\left\{j: v_{\tau j} \geq v_{\kappa j}\right\} .
$$

The discordance set contains the index of all parameters for which the alternative $x_{\tau}$ is worse than the alternative $x_{\kappa}$. This set can be described as follows.

$$
\text { For } \tau, \kappa \in \mathcal{I}_{m} \text { and } \tau \neq \kappa \text { : }
$$

$$
\mathcal{J}_{\tau \kappa}^{-}=\left\{j: v_{\tau j}<v_{\kappa j}\right\} .
$$

In other words, this set can be considered as the complement of the concordance set $\mathcal{J}_{\tau \kappa}^{+}$, i.e., $\mathcal{J}_{\tau \kappa}^{-}=$ $\mathcal{J} \backslash \mathcal{J}_{\tau \kappa}^{+}$where $\mathcal{J}=\left\{j: p_{j} \in \mathcal{P}\right\}$.

Step 7. The concordance matrix and discordance matrix are generated by employing the sets of concordance and discordance, respectively.

The concordance matrix can be expressed as follows:

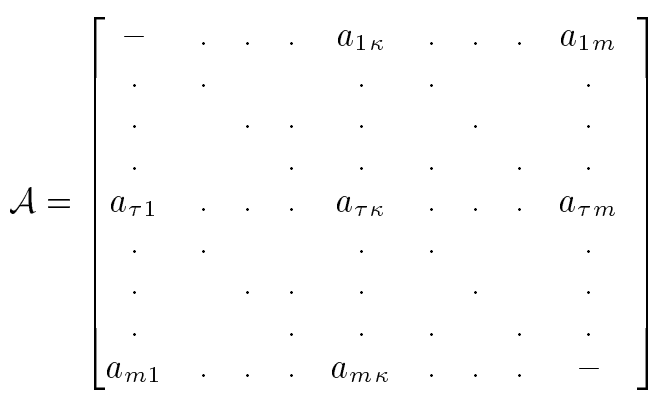

$$
\begin{aligned}
& =\left[a_{\tau \kappa}\right]_{m \times m},
\end{aligned}
$$

where: 


$$
a_{\tau \kappa}=\frac{\sum_{j \in \mathcal{J}_{\tau \kappa}^{+} \sum_{k=1}^{s} \omega_{j}^{k}}}{\sum_{j \in \mathcal{J}} \sum_{k=1}^{s} \omega_{j}^{k}}, \quad\left(0 \leq a_{\tau \kappa} \leq 1\right)
$$

for all $\tau, \kappa \in \mathcal{I}_{m}$. In other words, each component of concordance matrix is found as a summation of the (standardized) weights of all parameters corresponding to the indices in the concordance set $\mathcal{J}^{+}$.

The discordance matrix can be expressed as follows:

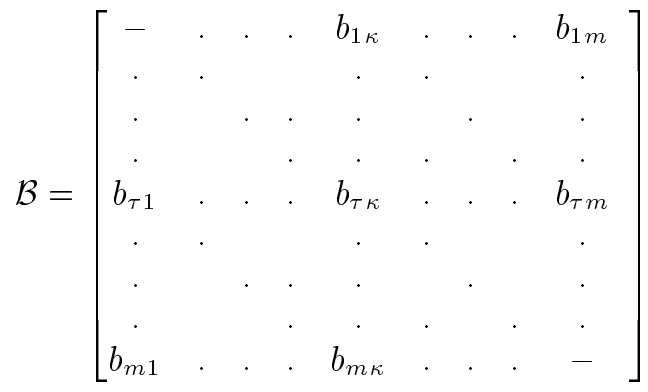

$$
\begin{aligned}
& =\left[b_{\tau \kappa}\right]_{m \times m},
\end{aligned}
$$

where:

$$
b_{\tau \kappa}=\frac{\sum_{j \in \mathcal{J}_{\tau \kappa}^{-}}\left|\mathrm{v}_{\tau j}-\mathrm{v}_{\kappa j}\right|}{\sum_{j \in \mathcal{J}}\left|\mathrm{v}_{\tau j}-\mathrm{v}_{\kappa j}\right|}, \quad\left(0 \leq b_{\tau \kappa} \leq 1\right)
$$

for all $\tau, \kappa \in \mathcal{I}_{m}$.

Step 8. The concordance threshold $\underline{\mathcal{A}}$ and discordance threshold $\underline{\mathcal{B}}$ are found.

The concordance threshold is calculated as follows:

$$
\underline{\mathcal{A}}=\frac{\sum_{\tau=1}^{m} \sum_{\kappa=1}^{m} a_{\tau \kappa}}{m(m-1)}, \quad(0 \leq \underline{\mathcal{A}} \leq 1),
$$

and the discordance threshold is calculated below:

$$
\underline{\mathcal{B}}=\frac{\sum_{\tau=1}^{m} \sum_{\kappa=1}^{m} b_{\tau \kappa}}{m(m-1)}, \quad(0 \leq \underline{\mathcal{B}} \leq 1) .
$$

Step 9. The effective concordance matrix $\mathcal{F}$ and effective discordance matrix $\mathcal{G}$ are created.

The effective concordance matrix $\mathcal{F}$ is measured based on the concordance threshold $\mathcal{A}$, as expressed in the following:

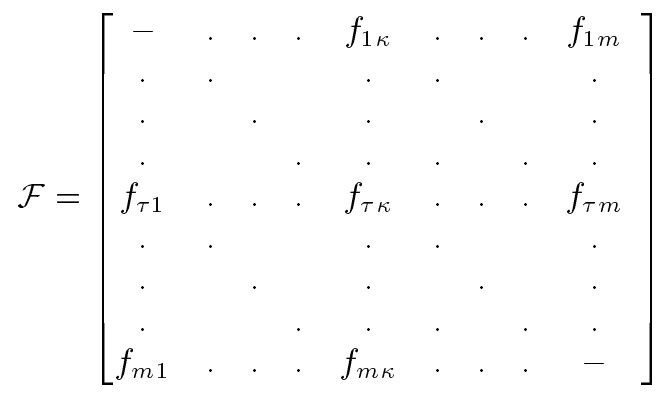

$$
\begin{aligned}
& =\left[f_{\tau \kappa}\right]_{m \times m},
\end{aligned}
$$

where:

$$
f_{\tau \kappa}=\left\{\begin{array}{lll}
1, & \text { if } a_{\tau \kappa} \geq \underline{\mathcal{A}} \\
0, & \text { if } a_{\tau \kappa}<\underline{\mathcal{A}}
\end{array}\right.
$$

The effective discordance matrix $\mathcal{G}$ is measured based on the discordance threshold $\mathcal{B}$, as expressed in the following:

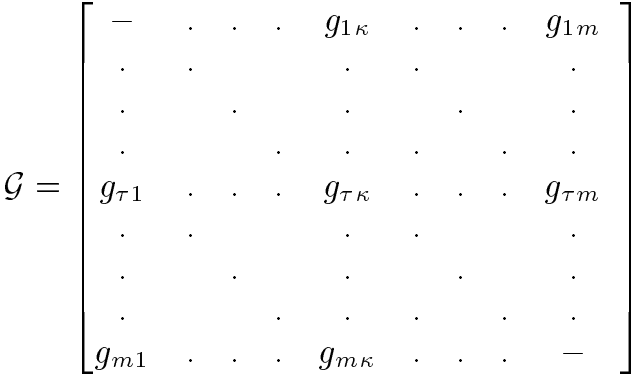

$$
\begin{aligned}
& =\left[g_{\tau \kappa}\right]_{m \times m},
\end{aligned}
$$

where:

$$
g_{\tau \kappa}= \begin{cases}0, & \text { if } b_{\tau \kappa}>\underline{\mathcal{B}} \\ 1, & \text { if } b_{\tau \kappa} \leq \underline{\mathcal{B}}\end{cases}
$$

Step 10. The aggregated outranking matrix $\mathcal{H}$ is constructed. Then, the aggregated outranking matrix $\mathcal{H}$ is established by merging the effective concordance information with effective discordance information. The matrix $\mathcal{H}$ can be described as follows:

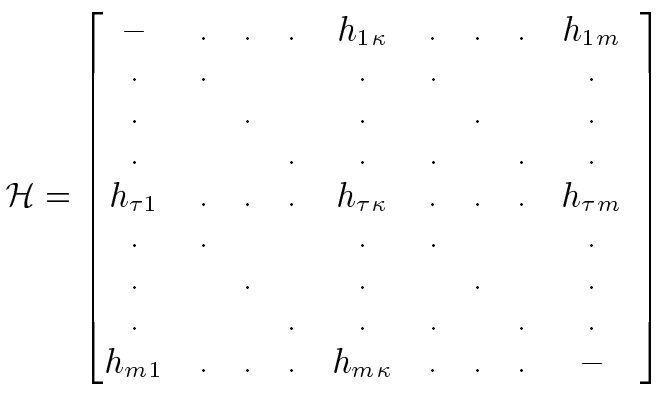

$$
\begin{aligned}
& =\left[h_{\tau \kappa}\right]_{m \times m},
\end{aligned}
$$

where:

$$
h_{\tau \kappa}=f_{\tau \kappa} \times g_{\tau \kappa},
$$

for all $\tau, \kappa \in \mathcal{I}_{m}$

Step 11. The alternatives (objects) are ranked in order of preference. The components in the aggregated outranking matrix $\mathcal{H}$ are indicative of the dominance of any alternative over the other. Therefore, according to this priority, a choice priority among the alternatives is considered to rank the alternatives.

Given the aggregated outranking matrix $\mathcal{H}$, the binary relations among the alternatives may take place as one of the following three situations:

(a) $x_{\tau} \succ x_{\kappa}$ (i.e., $x_{\tau}$ is strictly preferred over $x_{\kappa}$ or $x_{\tau}$ is dominant over $x_{\kappa}$ ) if $h_{\tau \kappa}=1$ and $h_{\kappa \tau}=0$; 
(b) $x_{\tau} \approx x_{\kappa}$ (i.e., $x_{\tau}$ is indifferent to $x_{\kappa}$ ) if $h_{\tau \kappa}=1$ and $h_{\kappa \tau}=1$

(c) $x_{\tau} ? x_{\kappa}$ (i.e., $x_{\tau}$ and $x_{\kappa}$ are incomparable) if $h_{\tau \kappa}=$ 0 and $h_{\kappa \tau}=0$.

Therefore, the ranking order of alternatives can be interpreted.

For the implementation of this model, a solution that follows the multi-criteria group-decision-making problem is offered.

Example 6.1. Supplier selection is among the most important issues in the supply chain management area. In this regard, a numerical example of a supplier selection problem adopted from $[57,58]$ was taken into account. A high-technology company that manufactures electronic products aims to evaluate and choose a materials supplier. Assume that $\mathfrak{X}=\left\{x_{1}, x_{2}, x_{3}, x_{4}\right\}$ is a set of four suppliers chosen as candidates (alternatives). A single decision maker may not be able to accurately consider all relevant aspects during decisionmaking. Therefore, the company's leader decides to put together a decision committee to determine a suitable supplier. A committee of three decision makers (experts) is established containing: 1) Financial expert $\left(E_{1}\right)$, who evaluates alternatives in terms of cost and finance; 2) Quality control expert $\left(E_{2}\right)$, who evaluates alternatives in terms of quality and safety; and 3 ) Engineering expert $\left(E_{3}\right)$, who evaluates alternatives in terms of engineering and technical aspects.

Six, evaluation parameters are also considered: 1 ) performance $\left.\left(p_{1}\right), 2\right)$ cost control $\left.\left(p_{2}\right), 3\right)$ management audit $\left.\left(p_{3}\right), 4\right)$ service $\left.\left(p_{4}\right), 5\right)$ company reputation $\left(p_{5}\right)$, and 6$)$ quality $\left(p_{6}\right)$. To deal with this problem, the steps of Algorithm 4 are followed:

Step 1. Decision makers (experts) $E_{1}, E_{2}$, and $E_{3}$ make their decisions based on the parameter subsets $Q_{1}=\left\{p_{1}, p_{2}, p_{3}, p_{5}\right\}, Q_{2}=\left\{p_{1}, p_{2}, p_{3}, p_{5}, p_{6}\right\}$, and $Q_{3}=\left\{p_{1}, p_{2}, p_{4}, p_{5}, p_{6}\right\}$, respectively. In addition, they measure the weights of their choice parameters, as shown in Table 16.

Decision makers (experts) present their opinion about the truth of alternative $x_{i}$ under the parameter $p_{j}$ and construct Table 17 .

Step 2. The (three-valued) decision matrices $\mathfrak{D}^{k}$ $(k=1,2,3)$ are constructed as follows:

$$
\begin{aligned}
\mathfrak{D}^{1} & =\left[\begin{array}{cccccc}
1 & 0.25 & 0 & 0 & 1 & 0 \\
0.25 & 1 & 1 & 0 & 0 & 0 \\
0 & 1 & 1 & 0 & 0.25 & 0 \\
0.25 & 0.25 & 0.25 & 0 & 1 & 0
\end{array}\right], \\
\mathfrak{D}^{2} & =\left[\begin{array}{cccccc}
0 & 0.125 & 1 & 0 & 0.125 & 0 \\
1 & 0 & 0 & 0 & 0 & 1 \\
1 & 1 & 1 & 0 & 0.125 & 0 \\
0.125 & 0 & 0.125 & 0 & 0 & 1
\end{array}\right], \\
\mathfrak{D}^{3} & =\left[\begin{array}{ccccccc}
0.25 & 0 & 0 & 0 & 0.25 & 0 \\
0 & 0.25 & 0 & 0 & 0.25 & 0 \\
1 & 0 & 0 & 0 & 0.25 & 1 \\
1 & 1 & 0 & 1 & 0.25 & 0
\end{array}\right] .
\end{aligned}
$$

Steps 3 and 4. For the decision matrices $\mathfrak{D}^{k}(k=$ $1,2,3)$, the normalized decision matrices $\mathfrak{R}^{k}(k=$ $1,2,3)$ and weighted normalized decision matrices $\mathrm{V}^{k}$ $(k=1,2,3)$ are constructed similar to that in Steps 3 and 4 of Example 5.1; hence, it is omitted.

Table 16. The weights of the decision maker's choice parameters.

\begin{tabular}{|c|c|c|c|c|c|c|c|c|c|c|c|c|c|c|c|c|c|c|}
\hline \multirow{2}{*}{$\begin{array}{c}\text { Decision makers: } \\
\mathfrak{X} / \mathcal{P}\end{array}$} & \multicolumn{6}{|c|}{$\begin{array}{l}\text { Financial expert } \\
\left(E_{1}\right)\end{array}$} & \multicolumn{6}{|c|}{$\begin{array}{l}\text { Quality control expert } \\
\qquad\left(E_{2}\right)\end{array}$} & \multicolumn{6}{|c|}{$\begin{array}{l}\text { Engineering expert } \\
\left(E_{3}\right)\end{array}$} \\
\hline & $p_{1}$ & $p_{2}$ & $p_{3}$ & $p_{4}$ & $p_{5}$ & $p_{6}$ & $p_{1}$ & $p_{2}$ & $p_{3}$ & $p_{4}$ & $p_{5}$ & $p_{6}$ & $p_{1}$ & $p_{2}$ & $p_{3}$ & $p_{4}$ & $p_{5}$ & $p_{6}$ \\
\hline$x_{1}$ & 1 & $\frac{1}{2}$ & 0 & 0 & 1 & 0 & 0 & $\frac{1}{2}$ & 1 & 0 & $\frac{1}{2}$ & 0 & $\frac{1}{2}$ & 0 & 0 & 0 & $\frac{1}{2}$ & 0 \\
\hline$x_{2}$ & $\frac{1}{2}$ & 1 & 1 & 0 & 0 & 0 & 1 & 0 & 0 & 0 & 0 & 1 & 0 & $\frac{1}{2}$ & 0 & 0 & $\frac{1}{2}$ & 0 \\
\hline$x_{3}$ & 0 & 1 & 1 & 0 & $\frac{1}{2}$ & 0 & 1 & 1 & 1 & 0 & $\frac{1}{2}$ & 0 & 1 & 0 & 0 & 0 & $\frac{1}{2}$ & 1 \\
\hline$x_{4}$ & $\frac{1}{2}$ & $\frac{1}{2}$ & $\frac{1}{2}$ & 0 & 1 & 0 & $\frac{1}{2}$ & 0 & $\frac{1}{2}$ & 0 & 0 & 1 & 1 & 1 & 0 & 1 & $\frac{1}{2}$ & 0 \\
\hline Impact coefficient $\xi_{k}$ : & \multicolumn{6}{|c|}{$\xi_{1}=2$} & \multicolumn{6}{|c|}{$\xi_{2}=3$} & \multicolumn{6}{|c|}{$\xi_{3}=2$} \\
\hline
\end{tabular}

\begin{tabular}{lccccccc}
\hline Decision makers/weights & $\boldsymbol{\omega}_{\mathbf{1}}$ & $\boldsymbol{\omega}_{\mathbf{2}}$ & $\boldsymbol{\omega}_{\mathbf{3}}$ & $\boldsymbol{\omega}_{\mathbf{4}}$ & $\boldsymbol{\omega}_{\mathbf{5}}$ & $\boldsymbol{\omega}_{\mathbf{6}}$ & \\
\hline Engineering expert $\left(E_{1}\right)$ & 0.2 & 0.15 & 0.5 & 0 & 0.15 & 0 & $\sum_{j=1}^{6} \omega_{j}^{1}=1$ \\
Financial expert $\left(E_{2}\right)$ & 0.15 & 0.25 & 0.25 & 0 & 0.1 & 0.25 & $\sum_{j=1}^{6} \omega_{j}^{2}=1$ \\
Quality control expert $\left(E_{3}\right)$ & 0.2 & 0.2 & 0 & 0.1 & 0.1 & 0.4 & $\sum_{j=1}^{6} \omega_{j}^{3}=1$ \\
\hline
\end{tabular}

Table 17. The expert's three valued soft sets $T_{Q_{1}}, T_{Q_{2}}$, and $T_{Q_{3}}$. 
Table 18. Sets of concordance and discordance.

\begin{tabular}{|c|c|c|c|c|c|c|c|c|}
\hline & \multicolumn{4}{|c|}{ Concordance set $\left(\mathcal{J}^{+}\right)$} & \multicolumn{4}{|c|}{ Discordance set $(\mathcal{J})$} \\
\hline & $x_{1}$ & $x_{2}$ & $x_{3}$ & $x_{4}$ & $x_{1}$ & $x_{2}$ & $x_{3}$ & $x_{4}$ \\
\hline$x_{1}$ & - & $\{1,4,5\}$ & $\{4,5\}$ & $\{1,3,5\}$ & - & $\{2,3,6\}$ & $\{1,2,3,6\}$ & $\{2,4,6\}$ \\
\hline$x_{2}$ & $\{2,3,4,6\}$ & - & $\{4\}$ & $\{3,6\}$ & $\{1,5\}$ & - & $\{1,2,3,5,6\}$ & $\{1,2,4,5\}$ \\
\hline$x_{3}$ & $\{1,2,3,4,6\}$ & $\{1,2,3,4,5,6\}$ & - & $\{1,2,3,6\}$ & $\{5\}$ & $\emptyset$ & - & $\{4,5\}$ \\
\hline$x_{4}$ & $\{2,4,6\}$ & $\{1,2,4,5,6\}$ & $\{4,5\}$ & - & $\{1,3,5\}$ & $\{3\}$ & $\{1,2,3,6\}$ & - \\
\hline
\end{tabular}

Step 5. Then, the average weighted normalized decision matrix is:

$$
\begin{aligned}
& \mathcal{V}=\mathrm{V}^{1} \oplus \mathrm{V}^{2} \oplus \mathrm{V}^{3}= \\
& {\left[\begin{array}{cccccc}
0.0744 & 0.0189 & 0.0586 & 0 & 0.0917 & 0 \\
0.0509 & 0.0504 & 0.116 & 0 & 0.0333 & 0.0589 \\
0.0816 & 0.117 & 0.1747 & 0 & 0.0656 & 0.1333 \\
0.0665 & 0.0732 & 0.0363 & 0.0333 & 0.0681 & 0.0589
\end{array}\right]} \\
& =\left[v_{i j}\right]_{4 \times 6},
\end{aligned}
$$

where the operation $\oplus$ represents the arithmetic mean.

Step 6. With the consideration of the average weighted normalized decision matrix $\mathcal{V}$, the concordance set and discordance set are determined and presented in Table 18.

Step 7. Then, the concordance matrix and discordance matrix are respectively generated as follows:

$$
\begin{aligned}
\mathcal{A} & =\left[\begin{array}{cccc}
- & 0.3333 & 0.15 & 0.55 \\
0.7 & - & 0.0333 & 0.4666 \\
0.8833 & 1 & - & 0.85 \\
0.45 & 0.75 & 0.15 & -
\end{array}\right] \\
& =\left[a_{\tau \kappa}\right]_{4 \times 4}, \quad \text { and } \\
\mathcal{B} & =\left[\begin{array}{cccc}
- & 0.6434 & 0.9314 & 0.7314 \\
0.3565 & - & 1 & 0.5719 \\
0.0685 & 0 & - & 0.1164 \\
0.2685 & 0.428 & 0.8718 & -
\end{array}\right]=\left[b_{\tau \kappa}\right]_{4 \times 4} .
\end{aligned}
$$

Step 8. The concordance threshold and discordance threshold are calculated as $\underline{\mathcal{A}}=0.5263$ and $\underline{\mathcal{B}}=$ 0.4989 , respectively.

Step 9. The effective concordance matrix $\mathcal{F}$ and effective discordance matrix $\mathcal{G}$ are created as follows:

$$
\begin{aligned}
\mathcal{F} & =\left[\begin{array}{cccc}
- & 0 & 0 & 1 \\
1 & - & 0 & 0 \\
1 & 1 & - & 1 \\
0 & 1 & 0 & -
\end{array}\right]=\left[f_{\tau \kappa}\right]_{4 \times 4}, \text { and } \\
\mathcal{G} & =\left[\begin{array}{cccc}
- & 0 & 0 & 0 \\
1 & - & 0 & 0 \\
1 & 1 & - & 1 \\
1 & 1 & 0 & -
\end{array}\right]=\left[g_{\tau \kappa}\right]_{4 \times 4} .
\end{aligned}
$$

Step 10. Then, the aggregated outranking matrix $\mathcal{H}$ is

$$
\mathcal{H}=\left[\begin{array}{cccc}
- & 0 & 0 & 0 \\
1 & - & 0 & 0 \\
1 & 1 & - & 1 \\
0 & 1 & 0 & -
\end{array}\right]=\left[h_{\tau \kappa}\right]_{4 \times 4}
$$

Step 11. Considering the aggregated outranking matrix $\mathcal{H}$, we obtain the following binary relations as:

- $h_{21}=1$ and $h_{12}=0 \Rightarrow x_{2} \succ x_{1}$,

- $h_{31}=1$ and $h_{13}=0 \Rightarrow x_{3} \succ x_{1}$,

- $h_{32}=1$ and $h_{23}=0 \Rightarrow x_{3} \succ x_{2}$,

- $h_{34}=1$ and $h_{43}=0 \Rightarrow x_{3} \succ x_{4}$,

- $h_{42}=1$ and $h_{24}=0 \Rightarrow x_{4} \succ x_{2}$.

Therefore, the ranking order of alternatives is found as $x_{3} \succ x_{4} \succ x_{2} \succ x_{1}$.

\section{Comparison and discussion}

Algorithm 2 is more general than Algorithm 1; in other words, it is the version that takes into account parameter weights. In this section, the performances of Algorithms 2, 3, and 4 are explained and evaluated. All of these algorithms can be used to deal with multicriteria group decision problems involving incomplete information. While each of them has a different operating philosophy, they also have one goal in common, that is, to combine the evaluations of multiple decisionmakers and to propose an optimal choice. They can also offer a choice according to the assessment of only one decision-maker. While Algorithm 2 can be applied if the decision-makers determine the same impact coefficient for "undetermined", there is no such limitation for Algorithms 3 and 4. The computational performance of each of our algorithms is critically analyzed by the experimental studies; hence, we have Table 19.

As shown in Table 19, the outputs of Algorithms 3 and 4 are the same. For many decision-making problems, the results obtained from these two algorithms either are identical or overlap each other. Moreover, all of these algorithms can be used for Examples 4.3 and 4.4 , and produce the same results. For the problems in 
Table 19. Comparing and matching the results of Algorithms 2, 3, and 4.

\begin{tabular}{clccc}
\hline & & \multicolumn{3}{c}{ Optimal choice } \\
\cline { 3 - 5 } Problems & Impact coefficients & Algorithm 2 & Algorithm 3 & Algorithm 4 \\
\hline Example 4.3 & $\xi=1$ (i.e., $\left.\xi_{1}=1, \xi_{2}=1\right)$ & $x_{3}$ & $x_{3}$ & $x_{3}$ \\
Example 4.4 & $\xi=1$ (i.e., $\left.\xi_{1}=1, \xi_{2}=1\right)$ & $x_{1}$ & $x_{1}$ & $x_{1}$ \\
Example 5.1 & $\xi_{1}=1.5, \xi_{2}=2$ & - & $x_{2}$ & $x_{2}$ \\
Example 6.1 & $\xi_{1}=2, \xi_{2}=3, \xi_{3}=2$ & - & $x_{3}$ & $x_{3}$ \\
\hline
\end{tabular}

Note: "-" means that the algorithm is not applicable to this problem.

Table 20. The comparison results of Algorithms 2, 3, and 4 with some existing soft decision making algorithms.

\begin{tabular}{cccccc}
\hline & & \multicolumn{4}{c}{ Optimal choice } \\
\cline { 3 - 6 } Ref. & Problem in the paper & $\begin{array}{c}\text { Algorithm in } \\
\text { the paper }\end{array}$ & Algorithm 2 Algorithm 3 & Algorithm 4 \\
\hline$[38]$ & $\begin{array}{c}\text { Application (Section 5) in [38] } \\
\text { (Example 5.17 in [35] }\end{array}$ & $u_{1}$ & $u_{1}$ & $u_{1}$ & $u_{1}$ \\
{$[35,36]$} & Example 3.3 in [36]) & $h_{1}, h_{2}, h_{3}$ & $h_{1}, h_{2}, h_{3}$ & $h_{1}$ & $h_{1} \succ h_{3} \succ h_{4} ?$ \\
{$[59]$} & Example 31 in [59] & $u_{3}$ & $u_{3}$ & $u_{3}$ & $h_{5} \& h_{2} \succ h_{4} ? h_{5}$ \\
{$[32]$} & Table 2 (Section 3.4) in [32] & $h_{1}, h_{6}$ & $h_{1}, h_{6}$ & $h_{1}, h_{6}$ & $u_{3}$ \\
\hline
\end{tabular}

Note: For Algorithms 2, 3, and 4, the weights of parameters in decision problem are taken equally and their sum is 1.

Examples 5.1 and 6.1 , the outputs of the algorithms coincide. These results support the efficiency and usefulness of the proposed algorithms.

Since the three-valued soft set is an extension of soft set, the emerging algorithms can be applied to decision-making problems based on the soft set(s). In this respect, the results of the proposed algorithms with those of some of the existing soft decision-making algorithms were compared. The details supporting this argument are presented in Table 20.

In this table, the weights of parameters in each of these problems were equally considered when making calculations in Algorithms 2, 3, and 4 (for instance, in application (Section 5) in [38], the parameter set is $X=\left\{x_{1}, x_{2}, x_{3}\right\}$ and so we specify $\omega_{1}=\omega_{2}=\omega_{3}=\frac{1}{3}$ ). Also, $\xi$ can be arbitrarily chosen in accordance with the comments on the selection of the impact coefficient mentioned above. It is clear that the arbitrary selection of $\xi$ will not change the result(s).

As shown in Table 20, the results of our algorithms coincide with those of existing soft decisionmaking algorithms. For Example 5.17 in [35] and Example 3.3 in [36], the optimal choice by the algorithms proposed in $[35,36]$ is $\left\{h_{1}, h_{2}, h_{3}\right\}$, while the optimal choice by Algorithm 3 is $h_{1}$ (where $h_{1} \succ$ $h_{3} \succ h_{2}$ ). This is not a contradiction, and this is the effect of normalizing the decision matrices in the model of TOPSIS (Algorithm 3). Considering the result of Algorithm 4 for the same problems, we say that $h_{1}$ and $h_{2}$ are incomparable, while $h_{3}$ and $h_{2}$ are incomparable. This does not contradict the result that the optimal choice is $\left\{h_{1}, h_{2}, h_{3}\right\}$, because what is certain is that $h_{1} \succ h_{4} ? h_{5}, h_{2} \succ h_{4} ? h_{5}$, and $h_{3} \succ h_{4}$ ? $h_{5}$. Consequently, the applicability of our algorithms to decision making based on both soft set and three-valued soft set demonstrates their performance range and advantages.

\section{Conclusion}

This study defined a three-valued soft set as a generalization of the soft set and its set-theoretic operations like intersection, union, difference, and symmetric difference. Moreover, the basic relationships concerning three-valued soft sets were described and the corresponding generalization of the operations on soft sets to these sets was highlighted. In this regard, some examples for them were provided. The algorithms supporting multi-criteria decision making for the threevalued soft set based on Technique for Order Preference by Similarity to Ideal Solution (TOPSIS) and ELimination Et Choice Translating (ELECTRE) techniques were formed and their outputs were compared. Thus, it was pointed out that these algorithms exhibited the applicability and efficiency of three-valued soft sets in handling the multi-criteria decision making involving uncertain or incomplete information.

We hope that this work will contribute to decision-making under uncertain and incomplete information in the context of soft sets and also will provide new ideas for future studies related to soft sets. Also, this study will motivate researchers to use three- 
valued logic stems in many practical applications such as data mining, data selection, data integration, data analysis, control of production processes, and pattern evaluation. In near future, we intend to explore new operations on three-valued soft sets and their practical applications in the fields such as science, social science, medical science, environmental science, economics, and so on.

\section{References}

1. Łukasiewicz, J. "Philosophische Bemerkungen zu mehrwertigen systemen des Aussagenkalkülls", Algebra C.R. Soc. Sci. Lett. Varsovie, 23, pp. 51-57 (1930).

2. Borowski, L. Ed., Selected Works of J. Eukasiewicz, North Holland Publishing Company, Amsterdam (1970).

3. Hájek, P., Metamathematics of Fuzzy Logic, Kluwer, Dordrecht (1998).

4. Kleene, S.C., Introduction to Metamathematics, North Holland Publishing Company, Amsterdam (1952).

5. Skolem, T.H. "A set theory based on a certain 3valued logic", Mathematica Scandinavica, 8, pp. 127136 (1960)

6. Zadeh, L.A. "Fuzzy sets", Information and Control, 8, pp. 338-353 (1965).

7. Pawlak, Z. "Rough sets", International Journal of Computer and Information Sciences, 11, pp. 341-356 (1982).

8. Molodtsov, D. "Soft set theory-first results", Computers and Mathematics with Applications, 37, pp. 19-31 (1999).

9. Maji, P.K., Biswas, R., and Roy, A.R. "Soft set theory", Computers and Mathematics with Applications, 45, pp. 555-562 (2003).

10. Ali, M.I., Feng, F., Liu, X., et al. "On some new operations in soft set theory", Computers and Mathematics with Applications, 57, pp. 1547-1553 (2009).

11. Aygün, E. and Kamacı, H. "Some generalized operations in soft set theory and their role in similarity and decision making", Journal of Intelligent and Fuzzy Systems, 36, pp. 6537-6547 (2019).

12. Sezgin, A. and Atagün, A.O. "On operations of soft sets", Computers and Mathematics with Applications, 61, pp. 1457-1467 (2011).

13. Kamacı, H. "Similarity measure for soft matrices and its applications", Journal of Intelligent and Fuzzy Systems, 36, pp. 3061-3072 (2019).

14. Zhu, P. and Wen, Q. "Operations of soft set revisited", Journal of Applied Mathematics, 2013, 7 pages (2013).

15. Gong, K., Xiao, Z., and Zhang, X. "The bijective soft set with its operations", Computers and Mathematics with Applications, 60, pp. 2270-2258 (2010).

16. Xiao, Z., Gong, K., Xia, S., et al. "Exclusive disjunctive soft sets", Computers and Mathematics with Applications, 59, pp. 2128-2137 (2010).
17. Karaaslan, F. and Karataş, S. "A new approach to bipolar soft sets and its applications", Discrete Mathematics, Algorithms and Applications, 7(4), 1550054 (2015).

18. Çetkin, V., Aygünog̃lu, A., and Aygün, H. "A new approach in handling soft decision making problems", Journal of Nonlinear Science and Applications, 9, pp. 231-239 (2016).

19. Khalil, A.M. and Hassan, N. "Inverse fuzzy soft set and its application in decision making", International Journal of Information and Decision Sciences, 11(1), pp. 73-92 (2019).

20. Abbasimehr, H. and Tarokh, M.J. "A novel interval type-2 fuzzy AHP-TOPSIS approach for ranking reviewers in online communities", Scientia Iranica, E, 23, pp. 2355-2373 (2016).

21. Adeel, A., Akram, M., and Koam, A.N.A. "Group decision-making based on $m$-polar hesitant fuzzy linguistic TOPSIS method", Symmetry, 11(6), 735 (2019).

22. Adeel, A., Akram, M., and Koam, A.N.A. "Multicriteria decision-making under $m$ HF ELECTRE-I and H $m$ F ELECTRE-I", Energies, 12(9), 1661 (2019).

23. Akram, M. and Adeel, A. "TOPSIS approach for MAGDM based on interval-valued hesitant fuzzy Nsoft environment", International Journal of Fuzzy Systems, 21(3), pp. 993-1009 (2019).

24. Akram, M., Adeel, A., and Alcantud, J.C.R. "Multicriteria group decision making using an $m$-polar hesitant fuzzy TOPSIS approach", Symmetry, 11(6), 795 (2019).

25. Garg, H. and Kaur, G. "Extended TOPSIS method for multi-criteria group decision-making problems under cubic intuitionistic fuzzy environment", Scientia Iranica, E, 25, pp. 1-18 (2018).

26. Jasemi, M. and Ahmadi, E. "A new fuzzy ELECTRE based multiple criteria method for personnel selection", Scientia Iranica, E, 25, pp. 943-953 (2018).

27. Garg, H., Agarwal, N., and Tripathi, A. "Some improved interactive aggregation operators under interval-valued intuitionistic fuzzy environment and its application to decision making process", Scientia Iranica, E, 24, pp. 2581-2604 (2017).

28. Garg, H. and Kumar, K. "A novel correlation coefficient of intuitionistic fuzzy sets based on the connection number of set pair analysis and its application", Scientia Iranica, E, 25, pp. 2373-2388 (2018).

29. Gitinavard, G., Mousavi, S.M., Vahdani, B., et al. "A new distance-based decision model in intervalvalued hesitant fuzzy setting for industrial selection problems", Scientia Iranica, E, 23, pp. 1928-1940 (2016).

30. Peng, X. "New operations for interval-valued Pythagorean fuzzy set", Scientia Iranica, E, 26, pp. 1049-1076 (2019). 
31. Tang, J. and Meng, F. "An approach to interval-valued intuitionistic fuzzy decision making based on induced generalized symmetrical Choquet Shapley operator", Scientia Iranica, E, 25, pp. 1456-1470 (2018).

32. Maji, P.K., Roy, A.R., and Biswas, R. "An application of soft sets in a decision making problem", Computers and Mathematics with Applications, 44, pp. 1077-1083 (2002).

33. Arora, D. and Garg, H. "Robust aggregation operators for multi-criteria decision-making with intuitionistic fuzzy soft environment", Scientia Iranica, E, 25(2), pp. 931-942 (2018).

34. Arora, R. and Garg, H. "Prioritized averaging/geometric aggregation operators under the intuitionistic fuzzy soft set environment", Scientia Iranica, E, 25, pp. 466-482 (2018).

35. Feng, F. and Li, Y. "Generalized uni-int decision making schemes based on choice value soft sets", European Journal of Operational Research, 220, pp. 162-170 (2012).

36. Han, B.-H. and Geng, S.-L. "Pruning method for optimal solutions of $i n t^{m}-i n t^{n}$ decision making", European Journal of Operational Research, 231, pp. 779-783 (2013).

37. Kamac1, H. "Selectivity analysis of parameters in soft set and its effect on decision making", International Journal of Machine Learning and Cybernetics, 11, pp. 313-324 (2020).

38. Eraslan, S. "A decision making method via TOPSIS on soft sets", Journal of New Results in Science, 8, pp. 57-71 (2015).

39. Avron, A. and Konikowska, B. "Rough sets and 3valued logics", Studia Logica, 90(1), pp. 69-92 (2008).

40. Çag̃man, N. and Enginog̃lu, S. "Soft set theory and uni-int decision making", European Journal of Operational Research, 207, pp. 848-855 (2010).

41. D'ottaviano, I.M.L. "The completeness and compactness of a three-valued first-order logic", Revista Colombiana de Matemáticas, 19, pp. 77-94 (1985).

42. Jacquette, D. "An internal determinacy metatheorem for Łukasiewicz's Aussagenkalküls", Bulletin of the Section of Logic, 29(3), pp. 115-124 (2000).

43. Ciucci, D. and Dubois, D. "A map of dependencies among three-valued logics", Information Sciences, 250, 162-177 (2013).

44. Cai, M., Li, Q., and Lang, G. "Shadowed sets of dynamic fuzzy sets", Granular Computing, 2, pp. 8594 (2017).

45. Pedrycz, W. "From fuzzy sets to shadowed sets: Interpretation and computing", International Journal of Intelligent Systems, 24(1), pp. 48-61 (2009).

46. Vogel, P., Read, R.W., Hansen, G.M., et al. "Congenital hydrocephalus in genetically engineered mice", Veterinary Pathology, 49(1), pp. 166-181 (2012).
47. Shih, H.-S., Shyur, H.-J., and Lee, E.S. "An extension of TOPSIS for group decision making", Mathematical and Computer Modelling, 45, pp. 801-813 (2007).

48. Hwang, C.L. and Yoon, K., Multiple Attribute Decision Making: Methods and Applications, New York: Springer-Verlag (1981).

49. Milani, A.S., Shanian, A., and Madoliat, R. "The effect of normalization norms in multiple attribute decision making models: A case study in gear material selection", Structural Multidisciplinary Optimization, 29(4), pp. 312-318 (2005).

50. Shih, H.-S., Lin, W.-Y., and Lee, E.S. "Group decision making for TOPSIS", Joint 9th IFSA World Congress and 20th NAFIPS International Conference, IFSA/NAFIPS, Vancouver, Canada, pp. 2712-2717 (2001).

51. Yoon, K.P. and Hwang, C.L., Multiple Attribute Decision Making: An Introduction, Sage Publishing Thousand Oaks, CA (1995).

52. Akram, M., Ilyas, F., and Garg, H. "Multicriteria group decision making based on ELECTRE I method in Pythagorean fuzzy information", Soft Computing, 24, pp. 3425-3453 (2020). https://doi.org/10.1007/s00500-019-04105-0

53. Alper, D. and Başdar, C. "A comparison of TOPSIS and ELECTRE methods: An application on the factoring industry", Business and Economics Research Journal, 8(3), pp. 627-646 (2017).

54. Birgün, S. and Cihan, E. "Supplier selection process using ELECTRE method", IEEE 2010 International Conference on Intelligent Systems and Knowledge Engineering, Hangzhou, China, pp. 634-639 (2010). DOI: 10.1109/ISKE.2010.5680767

55. Chen, T.-Y. "An IVIF-ELECTRE outranking method for multiple criteria decision-making with intervalvalued intuitionistic fuzzy sets", Technological and Economic Development of Economy, 22(3), pp. 416452 (2016).

56. Devi, K. and Yadav, S.P. "A multicriteria intuitionistic fuzzy group decision making for plant location selection with ELECTRE method", The International Journal of Advanced Manufacturing Technology, 66, pp. 1219-1229 (2013).

57. Xu, J. and Shen, F. "A new outranking choice method for group decision making under Atanassov's intervalvalued intuitionistic fuzzy environment", KnowledgeBased Systems, 70, pp. 177-188 (2014).

58. Zhong, L. and Yao, L. "An ELECTRE I-based multicriteria group decision making method with interval type-2 fuzzy numbers and its application to supplier selection", Applied Soft Computing, 57, pp. 556-576 (2017).

59. Kharal, A. "Soft approximations and uni-int decision making", The Scientific World Journal, 2014, 7 pages (2014). 


\section{Biographies}

Eyüp Akçetin is an Associate Professor at the Department of Accounting and Financial Management, Seydikemer School of Applied Sciences of Mugla SitkıKocman University in Turkey. He received his MSc degrees in Logistics and Maritime Transport from Dokuz Eylül University in 2007 and received another MSc degree in Informatics and Computer Engineering from Sakarya University in 2014 Turkey. He received his $\mathrm{PhD}$ degrees in Maritime Economy and Maritime Business Management from Istanbul University in 2012 Turkey. His research interests include multi-criteria decision-making, game theory, business intelligence and data mining, gamification, system dynamics in business. He has contributed to these scientific topics with many articles, books, and book chapters.

Hüseyin Kamacı is a Research Assistant at the Mathematics Department in the Science and Arts Faculty of Yozgat Bozok University, Turkey. He received his MSc and PhD degrees in Mathematics from Bozok University, Yozgat, Turkey in 2014 and 2018, respectively. His research interests include mathematical logic, set theory, operational research, computational intelligence, and decision-making. He has many valuable publications on these issues in different scientific journals. 